\title{
Radical Addition Approach to Asymmetric Amine Synthesis: Design, Implementation, and Comparison of Chiral $N$-Acylhydrazones
}

\author{
Gregory K. Friestad ${ }^{\mathrm{a} *}$, Cristian Draghici ${ }^{\mathrm{b}}$, Mustapha Soukrib, and Jun Qin ${ }^{\mathrm{b}}$ \\ ${ }^{a}$ Department of Chemistry, University of Iowa, Iowa City, Iowa, 52242 \\ ${ }^{b}$ Department of Chemistry, University of Vermont, Burlington, Vermont, 05405
}

\section{Supporting Information}

\section{Table of Contents}

$\begin{array}{ll}\text { Materials and Methods } & \text { S2 }\end{array}$

Preparation and Analysis of Hydrazones (Compounds 3-8) S2

Radical Addition to Hydrazones (Compounds 9-12) S5

$\begin{array}{ll}\text { Stereochemical Proofs (Compounds 13-15) } & \text { S11 }\end{array}$

Acetone Hydrazone (Compound 16) S13

$\begin{array}{ll}\text { References } & \text { S13 }\end{array}$

${ }^{1} \mathrm{H}$ NMR Spectra of 9aK, 10aE, 11aC, and $\mathbf{1 6} \quad \mathrm{S} 14$

NMR Spectra of 2a, 13, and $\mathbf{1 5}$ (for originals, see reference 16) S19 
Materials and Methods. Reactions employed oven- or flame-dried glassware under nitrogen unless otherwise noted. THF and toluene were distilled from sodium/benzophenone ketyl under argon. $\mathrm{CH}_{2} \mathrm{Cl}_{2}$ was distilled from $\mathrm{CaH}_{2}$ under argon or nitrogen. Nitrogen was passed successively through columns of anhydrous $\mathrm{CaSO}_{4}$ and R3-11 catalyst for removal of water and oxygen, respectively. All other materials were used as received or purified by standard procedures. Thin layer chromatography (TLC) employed glass $0.25 \mathrm{~mm}$ silica gel plates with UV indicator. Flash chromatography columns were packed with 230-400 mesh silica gel as a slurry in the initial elution solvent. Gradient flash chromatography was conducted by adsorption of product mixtures on silica gel, packing over a short pad of clean silica gel as a slurry in hexane, and eluting with a continuous gradient from hexane to the indicated solvent. Radial chromatography refers to centrifugally accelerated thin-layer chromatography performed with a Chromatotron using commercially supplied rotors. Melting points are uncorrected. Nuclear magnetic resonance (NMR) data were obtained at operating frequencies of $500 \mathrm{MHz}\left({ }^{1} \mathrm{H}\right)$ and $125 \mathrm{MHz}\left({ }^{13} \mathrm{C}\right)$. Infrared spectra were recorded using a single beam FT-IR spectrophotometer by standard transmission methods or by use of an attenuated total reflectance (ATR) probe. Optical rotations were determined using a digital polarimeter operating at ambient temperature. Low resolution mass spectra were obtained using sample introduction by dip, liquid chromatography or gas chromatography. High resolution mass spectra and combustion analyses were obtained from external commercial and institutional services. Chromatographic diastereomer ratio analyses employed GCMS with $15 \mathrm{~mL} x$ 0.25 mm I.D X $0.25 \mu$ F.T 5\%-phenyl-95\%-dimethylsiloxane column and helium as mobile phase or HPLC with Microsorb-MV Si 8um 100A or Chiralcel OD columns (2-propanol/hexane as mobile phase) or Chirex 3014 column (chloroform/hexane as mobile phase).

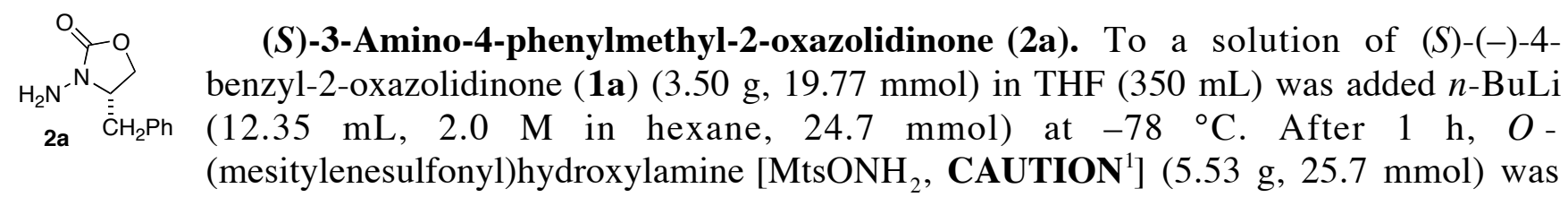
added, and the reaction mixture was allowed to warm to room temperature. Concentration and gradient flash chromatography (hexane $\rightarrow$ 1:5 hexane/EtOAc) afforded 2a as a pale yellow oil (2.85 $\mathrm{g}, 75 \%$ yield); $[\alpha]_{\mathrm{D}}^{22}+72.8^{\circ}\left(c\right.$ 3.7, $\left.\mathrm{CHCl}_{3}\right)$; IR (film) 3339, 3215, 3061, 2921, 1762, 1625, 1454, 1230, 1091, 1025, $921 \mathrm{~cm}^{-1}$; ${ }^{1} \mathrm{H}$ NMR $\left(500 \mathrm{MHz}, \mathrm{CDCl}_{3}\right) \delta 7.30(\mathrm{dd}, J=7.3,7.3 \mathrm{~Hz}, 2 \mathrm{H}), 7.23(\mathrm{dd}, J=7.3,7.3 \mathrm{~Hz}$, $1 \mathrm{H}), 7.14(\mathrm{~d}, J=7.3 \mathrm{~Hz}, 2 \mathrm{H}), 4.14(\mathrm{dd}, J=7.5,7.5 \mathrm{~Hz}, 1 \mathrm{H}), 3.98-3.90(\mathrm{~m}, 4 \mathrm{H}), 3.27(\mathrm{dd}, J=13.8,3.6$ $\mathrm{Hz}, 1 \mathrm{H}), 2.70(\mathrm{dd}, J=13.6,8.7 \mathrm{~Hz}, 1 \mathrm{H}) ;{ }^{13} \mathrm{C} \mathrm{NMR}\left(125 \mathrm{MHz}, \mathrm{CDCl}_{3}\right) \delta 159.1,135.5,129.0,128.7$, 126.9, 66.0, 59.5, 37.3; MS (CI) $\mathrm{m} / \mathrm{z}$ (relative intensity) $193\left([\mathrm{M}+\mathrm{H}]^{+}, 100 \%\right), 178(100 \%)$; Anal. Calcd for $\mathrm{C}_{10} \mathrm{H}_{12} \mathrm{~N}_{2} \mathrm{O}_{2}$ : C, 62.49; H, 6.29; N, 14.57. Found: C, 62.05; H, 6.34; N, 14.27. ${ }^{2}$

Preparation of Hydrazones: General Procedure A: To a solution of $\mathbf{1}$ in THF $(0.06 \mathrm{M})$ was added $n$-BuLi $(2.5 \mathrm{M}$ in hexane, $1.1 \mathrm{eq})$ at $-78{ }^{\circ} \mathrm{C}$. After $40 \mathrm{~min}, \mathrm{MtsONH}_{2}{ }^{1}(1.2 \mathrm{eq})$ was added, the reaction mixture was allowed to warm to room temperature, and the appropriate aldehyde (5 eq) was introduced. When the reaction was complete (TLC) the mixture was diluted with twice its volume of ether and partitioned between ether and water. The organic phase was dried over $\mathrm{Na}_{2} \mathrm{SO}_{4}$ and concentrated. Gradient flash chromatography (hexane $\rightarrow 3: 1$ hexane/EtOAc) furnished pure hydrazones as single $\mathrm{C}=\mathrm{N}$ isomers (>98:2, ${ }^{1} \mathrm{H}$ NMR). (Note: Although this General Procedure A was used to obtain 4-8 as described below, we now recommend the alternative General Procedure B.) 
Preparation of Hydrazones: General Procedure B. As described in detail elsewhere, ${ }^{3}$ hydrazones 3b-3e were prepared by the following sequence: (i) $\mathrm{NaH}$ (or $\mathrm{KH} ; 1.05$ equiv), dioxane, reflux; (ii) $O$ ( $p$-nitrobenzoyl)hydroxylamine $\left(\mathrm{NbzONH}_{2} ; 1.05\right.$ equiv); (iii) aldehyde, $p$ - $\mathrm{TsOH}$, toluene.)

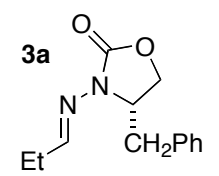

(S)-3-(Propylidene)amino-4-phenylmethyl-2-oxazolidinone (3a). From 1a (400 $\mathrm{mg}, 2.26 \mathrm{mmol})$ and propionaldehyde by General Procedure A was obtained 3a (425 mg, $81 \%$ yield) as a pale yellow oil; $[\alpha]_{\mathrm{D}}{ }^{25}+7.5^{\circ}\left(c 3.8, \mathrm{CHCl}_{3}\right)$; IR (film) $3062,2935,2877$, 1762, 1632, 1478, 1405, 1208, 1093, $930 \mathrm{~cm}^{-1} ;{ }^{1} \mathrm{H}$ NMR $\left(500 \mathrm{MHz}, \mathrm{CDCl}_{3}\right) \delta 7.98(\mathrm{t}, J=$ $5.2 \mathrm{~Hz}, 1 \mathrm{H}), 7.27-7.11(\mathrm{~m}, 5 \mathrm{H}), 4.31$ (dddd, $J=8.2,8.2,4.0,4.0 \mathrm{~Hz}, 1 \mathrm{H}), 4.17(\mathrm{dd}, J=8.8,8.8 \mathrm{~Hz}$, $1 \mathrm{H}), 4.02(\mathrm{dd}, J=8.8,5.6 \mathrm{~Hz}, 1 \mathrm{H}), 3.16(\mathrm{dd}, J=13.9,3,7 \mathrm{~Hz}, 1 \mathrm{H}), 2.76(\mathrm{dd}, J=13.9,8.6 \mathrm{~Hz}, 1 \mathrm{H})$, 2.37-2.34 (m, 2H), $1.10(\mathrm{t}, J=7.5 \mathrm{~Hz}, 3 \mathrm{H}) ;{ }^{13} \mathrm{C} \mathrm{NMR}\left(125 \mathrm{MHz}, \mathrm{CDCl}_{3}\right) \delta 157.3,154.3,135.2,129.1$, 128.7, 127.0, 65.6, 57.6, 37.1, 26.5, 10.5; MS (CI) m/z (relative intensity) $233\left([\mathrm{M}+\mathrm{H}]^{+}, 100 \%\right), 232$ (M, 7\%); Anal. Calcd for $\mathrm{C}_{13} \mathrm{H}_{16} \mathrm{~N}_{2} \mathrm{O}_{2}$ : C, 67.22; H, 6.94; N, 12.06. Found: C, 66.98; H, 6.83; N, 12.06.

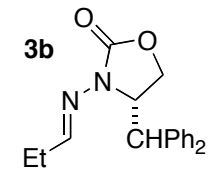

(S)-3-(Propylidene)amino-4-diphenylmethyl-2-oxazolidinone (3b). From 1 b (160 $\mathrm{mg}, 1.03 \mathrm{mmol}$ ) and propionaldehyde by General Procedure B was obtained $\mathbf{3 b}(192 \mathrm{mg}$, $99 \%$ yield) as a pale yellow oil; $[\alpha]_{\mathrm{D}}{ }^{28}+32.3^{\circ}\left(c 0.65, \mathrm{CHCl}_{3}\right)$; IR (film) 3493, 2982, 2913, 1753, 1495, 1410, 1222, 1091, $1030 \mathrm{~cm}^{-1} ;{ }^{1} \mathrm{H}$ NMR $\left(500 \mathrm{MHz}, \mathrm{CDCl}_{3}\right) \delta 7.82(\mathrm{t}, J$ $=4.35 \mathrm{~Hz}, 1 \mathrm{H}), 7.21-7.04(\mathrm{~m}, 10 \mathrm{H}), 4.81(\mathrm{ddd}, J=8.5,6.1,6.1 \mathrm{~Hz}, 1 \mathrm{H}), 4.38(\mathrm{~d}, J=6.4 \mathrm{~Hz}, 1 \mathrm{H}), 4.33$ $(\mathrm{dd}, J=8.7,8.7 \mathrm{~Hz}, 1 \mathrm{H}), 4.06(\mathrm{dd}, J=9.0,5.6 \mathrm{~Hz}, 1 \mathrm{H}), 2.14-2.08(\mathrm{~m}, 2 \mathrm{H}), 0.86(\mathrm{t}, J=7.5 \mathrm{~Hz}, 3 \mathrm{H}) ;{ }^{13} \mathrm{C}$ NMR (500 MHz, $\left.\mathrm{CDCl}_{3}\right) \delta 159.2,154.7,140.2,139.1,128.8$ (2C), 128.7, 128.6, 127.4, 126.9, 64.9, 59.9, 53.1, 26.5, 10.2; MS (CI) $\mathrm{m} / z$ (relative intensity) $309.5\left([\mathrm{M}+\mathrm{H}]^{+}, 100 \%\right)$; Anal. Calcd for $\mathrm{C}_{19} \mathrm{H}_{20} \mathrm{~N}_{2} \mathrm{O}_{2}: \mathrm{C}, 74.00 ; \mathrm{H}, 6.54 ; \mathrm{N}, 9.08$. Found: $\mathrm{C}, 73.82 ; \mathrm{H}, 6.60 ; \mathrm{N}, 8.85$.

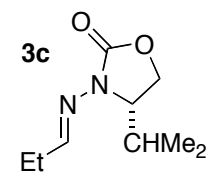

(S)-3-(Propylidene)amino-4-isopropyl-2-oxazolidinone (3c). From 1c (450 mg, $3.94 \mathrm{mmol}$ ) and propionaldehyde by General Procedure B was obtained 3c (511 $\mathrm{mg}, 84 \%$ yield) as a pale yellow oil. Spectroscopic data were in good agreement with those previously reported. ${ }^{4}$

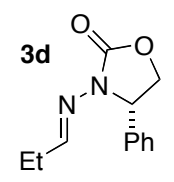

(S)-3-(Propylidene)amino-4-phenyl-2-oxazolidinone (3d). From 1d (164 mg, 0.92 $\mathrm{mmol}$ ) and propionaldehyde by General Procedure B was obtained 3d (153 mg, $76 \%$ yield) as a colorless oil; $[\alpha]_{\mathrm{D}}{ }^{22}+1029^{\circ}\left(c 0.55, \mathrm{CHCl}_{3}\right)$; IR (film) 3033, 2973, 1771, 1458, 1402, 1323, 1210, 1094, $1038 \mathrm{~cm}^{-1} ;{ }^{1} \mathrm{H}$ NMR $\left(500 \mathrm{MHz}, \mathrm{CDCl}_{3}\right) \delta 7.40-7.24(\mathrm{~m}, 5 \mathrm{H}), 7.14(\mathrm{t}, J=$ $5.4 \mathrm{~Hz}, 1 \mathrm{H}), 5.17(\mathrm{dd}, J=8.9,5.9 \mathrm{~Hz}, 1 \mathrm{H}), 4.73(\mathrm{t}, J=8.9 \mathrm{~Hz}, 1 \mathrm{H}), 4.11(\mathrm{dd}, J=8.9,5.8 \mathrm{~Hz}, 1 \mathrm{H}), 2.25-$ $2.19(\mathrm{~m}, 2 \mathrm{H}), 0.93(\mathrm{t}, J=7.6 \mathrm{~Hz}, 3 \mathrm{H}) ;{ }^{13} \mathrm{C} \mathrm{NMR}\left(500 \mathrm{MHz}, \mathrm{CDCl}_{3}\right) \delta 154.9,153.1,136.9,129.4,128.8$, 125.9, 69.5, 58.9, 26.2, 10.7; MS (CI) $\mathrm{m} / \mathrm{z}$ (relative intensity) $219.2\left([\mathrm{M}+\mathrm{H}]^{+}, 100 \%\right.$ ); Anal. Calcd for $\mathrm{C}_{12} \mathrm{H}_{14} \mathrm{~N}_{2} \mathrm{O}_{2}$ : C, 66.04; H, 6.47; N, 12.84. Found: C, 66.36; H, 6.64 ; N, 12.50.

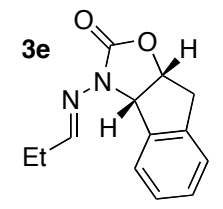

Propionaldehyde N-Acylhydrazone 3e. From 1 e $(243 \mathrm{mg}, 1.30 \mathrm{mmol})$ and propionaldehyde by General Procedure B was obtained 3e $(247 \mathrm{mg}, 83 \%$ yield $)$ as a pale yellow oil; $[\alpha]_{\mathrm{D}}{ }^{25}+118.0^{\circ}\left(c 0.7, \mathrm{CHCl}_{3}\right)$; IR (film) 2970, 1770, 1400, 1365, 1200, 1110, $1040 \mathrm{~cm}^{-1} ;{ }^{1} \mathrm{H}$ NMR $\left(500 \mathrm{MHz}, \mathrm{CDCl}_{3}\right) \delta 8.13(\mathrm{t}, J=5.0 \mathrm{~Hz}, 1 \mathrm{H}), 7.42(\mathrm{~d}, J=7.6 \mathrm{~Hz}$, $1 \mathrm{H}), 7.29-7.18(\mathrm{~m}, 3 \mathrm{H}), 5.47(\mathrm{~d}, J=7.2 \mathrm{~Hz}, 1 \mathrm{H}), 5.22(\mathrm{ddd}, J=7.0,7.0,1.3 \mathrm{~Hz}, 1 \mathrm{H})$, $3.28\left(\mathrm{ABX}, \Delta \mathrm{v}_{\mathrm{AB}}=51.6 \mathrm{~Hz}, \mathrm{~J}_{\mathrm{AB}}=17.9 \mathrm{~Hz}, \mathrm{~J}_{\mathrm{AX}}=6.5 \mathrm{~Hz}, \mathrm{~J}_{\mathrm{BX}}=0 \mathrm{~Hz}, 2 \mathrm{H}\right), 2.41-2.35(\mathrm{~m}, 2 \mathrm{H}), 1.14(\mathrm{t}, J=$ $7.6 \mathrm{~Hz}, 3 \mathrm{H}) ;{ }^{13} \mathrm{C} \mathrm{NMR}\left(500 \mathrm{MHz}, \mathrm{CDCl}_{3}\right) \delta 154.6,153.3,140.2,138.4,129.5,127.5,126.0,125.4,77.1$, 64.3, 38.4, 26.5, 10.6; MS (CI) $\mathrm{m} / \mathrm{z}$ (relative intensity) $231.5\left([\mathrm{M}+\mathrm{H}]^{+}, 100 \%\right)$; Anal. Calcd for $\mathrm{C}_{13} \mathrm{H}_{14} \mathrm{~N}_{2} \mathrm{O}_{2}$ : C, 67.81; H, 6.13; N, 12.17. Found: C, 67.63; H, 6.17; N, 11.95. 
(S)-3-(2'-Methylpropylidene)amino-4-phenylmethyl-2-oxazolidinone (4). From $1 \mathrm{a}$ (300 mg, 1.69

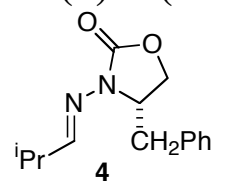
mmol) and isobutyraldehyde by General Procedure A was obtained 4 (291 mg, 70\% yield) as a colorless solid; $\mathrm{mp} 43-45^{\circ} \mathrm{C} ;[\alpha]_{\mathrm{D}}^{30}+9.6^{\circ}\left(c 2.1, \mathrm{CHCl}_{3}\right)$; IR (film) 3086 , 3029, 2929, 2965, 1762, 1631, 1604, 1497, 1454, 1404, 1289, 1210, 1101, 1007, $923 \mathrm{~cm}^{-}$ ${ }^{1} ;{ }^{1} \mathrm{H}$ NMR $\left(500 \mathrm{MHz}, \mathrm{CDCl}_{3}\right) \delta 7.97(\mathrm{~d}, J=6.6 \mathrm{~Hz}, 1 \mathrm{H}), 7.30(\mathrm{dd}, J=7.2,7.2 \mathrm{~Hz}, 2 \mathrm{H})$, $7.24(\mathrm{dd}, J=7.2,7.2 \mathrm{~Hz}, 1 \mathrm{H}), 7.14(\mathrm{~d}, J=7.6 \mathrm{~Hz}, 2 \mathrm{H}), 4.35-4.30(\mathrm{~m}, 1 \mathrm{H}), 4.21(\mathrm{dd}, J=8.8,8.8 \mathrm{~Hz}$, $1 \mathrm{H}), 4.05(\mathrm{dd}, J=8.7,5.6 \mathrm{~Hz}, 1 \mathrm{H}), 3.20(\mathrm{dd}, J=13.8,3.6 \mathrm{~Hz}, 1 \mathrm{H}), 2.80(\mathrm{dd}, J=13.8,8.7 \mathrm{~Hz}, 1 \mathrm{H}), 2.60$ $(\mathrm{m}, 1 \mathrm{H}), 1.14(\mathrm{~d}, J=6.9 \mathrm{~Hz}, 6 \mathrm{H}) ;{ }^{13} \mathrm{C} \mathrm{NMR}\left(125 \mathrm{MHz}, \mathrm{CDCl}_{3}\right) \delta 161.3,154.4,135.4,129.2,128.8$, 127.1, 65.6, 57.9, 37.3, 32.2, 19.73, 19.65; MS (CI) m/z (relative intensity) $247\left([\mathrm{M}+\mathrm{H}]^{+}, 100 \%\right), 246$ (M, 21\%); Anal. Calcd for $\mathrm{C}_{14} \mathrm{H}_{18} \mathrm{~N}_{2} \mathrm{O}_{2}$ : C, 68.27; H, 7.37; N, 11.37. Found: C, 68.03; H, 7.37; N, 11.43.

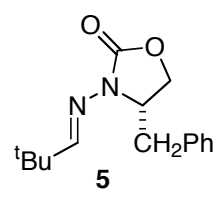

(S)-3-(2,2-Dimethylpropylidene)amino-4-phenylmethyl-2-oxazolidinone (5). To a solution of $\mathbf{2 a}(180 \mathrm{mg}, 0.94 \mathrm{mmol})$ in toluene $(10 \mathrm{~mL})$ was added trimethylacetaldehyde $(0.98 \mathrm{~mL}, 9.37 \mathrm{mmol})$ and a catalytic amount of $p$-toluenesulfonic acid. The mixture was stirred overnight at room temperature. Concentration and gradient flash chromatography (hexane $\rightarrow$ 3:1 hexane/EtOAc) afforded 5 (235 $\mathrm{mg}, 96 \%$ yield, 72\% yield for two steps from 1) as a colorless solid; $\mathrm{mp} 90.5-91.5^{\circ} \mathrm{C} ;[\alpha]_{\mathrm{D}}{ }^{26}+11.7^{\circ}\left(c 1.6, \mathrm{CHCl}_{3}\right)$; IR (film) $3060,3030,2954$, 2867, 1760, 1629, 1498, 1434, 1417, 1248, 1088, 997, 922, $846 \mathrm{~cm}^{-1} ;{ }^{1} \mathrm{H}$ NMR (500 MHz, $\left.\mathrm{CDCl}_{3}\right)$ $\delta 8.05(\mathrm{~s}, 1 \mathrm{H}), 7.31(\mathrm{dd}, J=7.6,7.6 \mathrm{~Hz}, 2 \mathrm{H}), 7.25(\mathrm{dd}, J=7.6,7.6 \mathrm{~Hz}, 1 \mathrm{H}), 7.15(\mathrm{~d}, J=7.4 \mathrm{~Hz}, 2 \mathrm{H})$, $4.37-4.31(\mathrm{~m}, 1 \mathrm{H}), 4.22(\mathrm{dd}, J=8.8,8.8 \mathrm{~Hz}, 1 \mathrm{H}), 4.05(\mathrm{dd}, J=8.7,5.9 \mathrm{~Hz}, 1 \mathrm{H}), 3.18(\mathrm{dd}, J=13.8,3.6$ $\mathrm{Hz}, 1 \mathrm{H}), 2.83(\mathrm{dd}, J=13.8,8.5 \mathrm{~Hz}, 1 \mathrm{H}), 1.15(\mathrm{~s}, 9 \mathrm{H}) ;{ }^{13} \mathrm{C} \mathrm{NMR}\left(125 \mathrm{MHz}, \mathrm{CDCl}_{3}\right) \delta 163.8,154.3$, 135.4, 129.2, 128.7, 127.0, 65.5, 58.0, 37.3, 35.4, 27.2; MS (CI) $\mathrm{m} / \mathrm{z}$ (relative intensity) $261\left([\mathrm{M}+\mathrm{H}]^{+}\right.$, $100 \%$ ); Anal. Calcd for $\mathrm{C}_{15} \mathrm{H}_{20} \mathrm{~N}_{2} \mathrm{O}_{2}$ : C, 69.20; H, 7.74; N, 10.76. Found: C, 69.34; H, 7.72; N, 10.79.

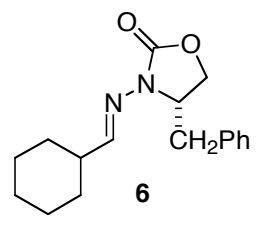

(S)-3-(Cyclohexylmethylidene)amino-4-phenylmethyl-2-oxazolidinone (6). From $1 \mathrm{a}(600 \mathrm{mg}, 3.39 \mathrm{mmol})$ and cyclohexanecarboxaldehyde by General Procedure A was obtained $6(630 \mathrm{mg}, 65 \%$ yield $)$ as a colorless solid; $\mathrm{mp} 53-55^{\circ} \mathrm{C}$; $[\alpha]_{\mathrm{D}}{ }^{25}+0.98^{\circ}$ (c 2.4, $\mathrm{CHCl}_{3}$ ); IR (film) 3086, 3062, 2923, 2852, 1764, 1628, 1604, 1478, 1497, 1207 , 1088, 971, 916, $893 \mathrm{~cm}^{-1} ;{ }^{1} \mathrm{H}$ NMR $\left(500 \mathrm{MHz} \mathrm{CDCl}_{3}\right) \delta 7.90(\mathrm{~d}, J=11.5 \mathrm{~Hz}, 1 \mathrm{H}), 7.30(\mathrm{dd}, J=7.1,7.1$ $\mathrm{Hz}, 2 \mathrm{H}), 7.24(\mathrm{dd}, J=6.8,6.8 \mathrm{~Hz}, 1 \mathrm{H}), 7.13(\mathrm{~d}, J=7.1 \mathrm{~Hz}, 2 \mathrm{H}), 4.20(\mathrm{dd}, J=8.5,8.5 \mathrm{~Hz}, 1 \mathrm{H}), 4.05$ (dd, $J=8.7,5.6 \mathrm{~Hz}, 1 \mathrm{H}), 3.18(\mathrm{dd}, J=13.9,3.7 \mathrm{~Hz}, 1 \mathrm{H}), 2.79(\mathrm{dd}, J=13.9,8.7 \mathrm{~Hz}, 1 \mathrm{H}), 2.38-2.24(\mathrm{~m}, 1 \mathrm{H})$, 1.85-1.64 (m, 5H), 1.34-1.22 (m, 5H), 4.35-4.29 (m, 1H); $\left.{ }^{13} \mathrm{C} \mathrm{NMR} \mathrm{(125} \mathrm{MHz,} \mathrm{CDCl}_{3}\right) \delta 160.4,154.3$, 135.4, 129.2, 128.8, 127.2, 65.6, 57.8, 41.6, 37.2, 30.1, 30.0, 25.9, 25.4; MS (CI) $\mathrm{m} / z$ (relative intensity) $287\left([\mathrm{M}+\mathrm{H}]^{+}, 100 \%\right)$; Anal. Calcd for $\mathrm{C}_{17} \mathrm{H}_{22} \mathrm{~N}_{2} \mathrm{O}_{2}$ : C, 71.30; H, 7.74; N, 9.78. Found: C, 71.25; H, 7.80; N, 9.68 .

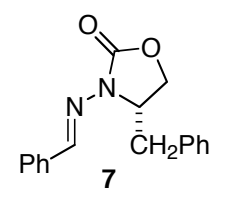

(S)-3-(Benzylidene)amino-4-phenylmethyl-2-oxazolidinone (7). To a solution of 2a (prepared from $3.56 \mathrm{mmol} \mathbf{1 a}$ and used without purification) in toluene $(70 \mathrm{~mL})$ was added benzaldehyde $(2.17 \mathrm{~mL}, 21.4 \mathrm{mmol})$ and a catalytic amount of p-toluenesulfonic acid. After heating at reflux for 1 hour, concentration and gradient flash chromatography (hexane $\rightarrow$ 3:1 hexane/EtOAc) afforded 7 (670 $\mathrm{mg}, 67 \%$ yield for two steps from 1a). Using General Procedure B, the yield was $80 \%$. $^{3}$ Colorless solid; mp 95.6-95.7 ${ }^{\circ} \mathrm{C}$; $[\alpha]_{\mathrm{D}}{ }^{25}-22.3^{\circ}\left(c 0.98, \mathrm{CHCl}_{3}\right) ; \mathrm{IR}^{2}$ (film) 3061, 3028, 2976, 2917, 1768, 1476, 1449, 1238, 1207, 1092, 940, $846 \mathrm{~cm}^{-1}$; ${ }^{1} \mathrm{H}$ NMR $(500 \mathrm{MHz}$, $\left.\mathrm{CDCl}_{3}\right) \delta 8.90(\mathrm{~s}, 1 \mathrm{H}), 7.75-7.72(\mathrm{~m}, 2 \mathrm{H}), 7.42-7.39(\mathrm{~m}, 3 \mathrm{H}), 7.32(\mathrm{dddd}, J=7.5,7.5,1.2,1.2 \mathrm{~Hz}, 2 \mathrm{H})$, 7.26 (dddd, $J=7.4,7.4,1.3,1.3 \mathrm{~Hz}, 1 \mathrm{H}), 7.19(\mathrm{ddd}, J=6.9,1.5,1.5 \mathrm{~Hz}, 2 \mathrm{H}), 4.47$ (ddd, $J=9.1,9.1$, $5.5,3.9 \mathrm{~Hz}, 1 \mathrm{H}), 4.30(\mathrm{dd}, J=8.8,8.8 \mathrm{~Hz}, 1 \mathrm{H}), 4.14(\mathrm{dd}, J=8.9,5.4 \mathrm{~Hz}, 1 \mathrm{H}), 3.31(\mathrm{dd}, J=13.9,3.8$ $\mathrm{Hz}, 1 \mathrm{H}), 2.92(\mathrm{dd}, J=13.9,8.6 \mathrm{~Hz}, 1 \mathrm{H}) ;{ }^{13} \mathrm{C} \mathrm{NMR}\left(125 \mathrm{MHz}, \mathrm{CDCl}_{3}\right) \delta 153.7,150.8,135.3,134.4$, 
Friestad et al.

130.4, 129.3, 128.9, 128.6, 127.4, 127.2, 65.7, 58.3, 37.7; MS (CI) m/z (relative intensity) $281\left([\mathrm{M}+\mathrm{H}]^{+}\right.$, $100 \%$ ); Anal. Calcd for $\mathrm{C}_{17} \mathrm{H}_{16} \mathrm{~N}_{2} \mathrm{O}_{2}$ : C, 72.84; H, 5.75; N, 9.99. Found: C, 72.91; H, 5.84; N, 9.98.

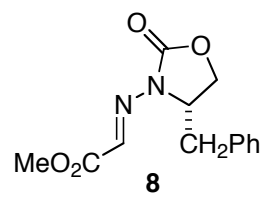

\section{(S)-3-(Methoxycarbonylmethylidene)amino-4-phenylmethyl-2-oxazolidinone}

(8). To a solution of 2a (prepared from $3.10 \mathrm{mmol} \mathbf{1 a}$ and used without purification) in toluene $(20 \mathrm{~mL})$ was added methyl glyoxylate $(300 \mathrm{mg}, 3.41 \mathrm{mmol})$ and a catalytic amount of $p$-toluenesulfonic acid. The mixture was heated at reflux for 1 hour. Concentration and gradient flash chromatography (hexane $\rightarrow 3: 1$ hexane/EtOAc) afforded 8 (580 mg, $71 \%$ yield for two steps from 1a) as a colorless oil; $[\alpha]_{\mathrm{D}}^{22}+32.9^{\circ}\left(c 0.9, \mathrm{CHCl}_{3}\right)$; IR (film) 3029, 2953, 1786, 1717, 1593, 1455, 1283, 1202, 1173, $913 \mathrm{~cm}^{-1} ;{ }^{1} \mathrm{H}$ NMR $\left(500 \mathrm{MHz}, \mathrm{CDCl}_{3}\right) \delta 8.40(\mathrm{~s}$, 1H), 7.31-7.14 (m, 5H), 4.47 (dddd, $J=8.4,8.4,4.8,3.8 \mathrm{~Hz}, 1 \mathrm{H}), 4.32$ (dd, $J=9.1,8.2 \mathrm{~Hz}, 1 \mathrm{H}), 4.18$ $(\mathrm{dd}, J=9.1,4.9 \mathrm{~Hz}, 1 \mathrm{H}), 3.87(\mathrm{~s}, 3 \mathrm{H}), 3.29(\mathrm{dd}, J=13.9,3.7 \mathrm{~Hz}, 1 \mathrm{H}), 2.88(\mathrm{dd}, J=13.9,8.9 \mathrm{~Hz}, 1 \mathrm{H})$; ${ }^{13} \mathrm{C} \mathrm{NMR}\left(125 \mathrm{MHz}, \mathrm{CDCl}_{3}\right) \delta 163.7,152.3,134.4,129.3,129.0,127.5,65.9,57.7,52.5,37.5 ; \mathrm{MS}$ (CI) $\mathrm{m} / \mathrm{z}$ (relative intensity) $263\left([\mathrm{M}+\mathrm{H}]^{+}, 85 \%\right), 231(100 \%)$; Anal. Calcd for $\mathrm{C}_{13} \mathrm{H}_{14} \mathrm{~N}_{2} \mathrm{O}_{4}: \mathrm{C}, 59.54 ; \mathrm{H}, 5.38$; N, 10.68. Found: C, 59.55; H, 5.47; N, 10.56.

Tin-Mediated Radical Addition to Hydrazones (Tables 2-4): General Procedure C. To a solution of the hydrazone in $\mathrm{CH}_{2} \mathrm{Cl}_{2}$ (ca. $0.25 \mathrm{M}$ ) was added $\mathrm{ZnCl}_{2}\left(1 \mathrm{M}\right.$ in $\mathrm{Et}_{2} \mathrm{O}, 2 \mathrm{eq}$ ). After $1 \mathrm{~h}$ at room temperature, the mixture was cooled to $-78{ }^{\circ} \mathrm{C}$, followed by addition of $\mathrm{Et}_{3} \mathrm{~B}$ (10 eq) and the appropriate alkyl iodide (10 eq). $\mathrm{Bu}_{3} \mathrm{SnH}\left(0.25 \mathrm{M}_{\text {in }} \mathrm{CH}_{2} \mathrm{Cl}_{2}, 5\right.$ eq) and $\mathrm{O}_{2}$ (ca. $7 \mathrm{~mL} / \mathrm{mmol}$ hydrazone) were introduced by syringe pump over ca. $5 \mathrm{~h}$. The reaction mixture was allowed to warm slowly to room temperature. After $2 \mathrm{~d}$, stannanes were removed by dilution with EtOAc, stirring overnight with excess KF, and filtration through a short pad of silica gel. Concentration and radial chromatography (hexane/EtOAc) afforded $N$-acylhydrazines 9-12. Side products 9aA and 9aB were obtained in varying amounts in some experiments. For those $N$-acylhydrazines for which diastereomer ratios are reported, control experiments established that no change in the diastereomer ratios occurred prior to analysis. Diastereomer ratios were determined by comparison with authentic diastereomer mixtures prepared by an alternative route. ${ }^{6}$ Diastereomer ratios of $9 \mathbf{a C}-9 \mathbf{a F}$ and $9 \mathbf{e C}$ were determined by HPLC. Diastereomer ratios of $N$-acylhydrazines $12 \mathbf{a C}-\mathbf{1 2 a F}$ were determined by GCMS. Diastereomer ratios of $9 \mathrm{bC}$ and $9 \mathrm{cC}$ were determined by integration of ${ }^{1} \mathrm{H}$ NMR data obtained in the presence of the NMR shift reagent tris $(6,6,7,7,8,8,8$-heptafluoro-2,2-dimethyl-3,5-octanedionato) europium(III) (Siever's reagent).

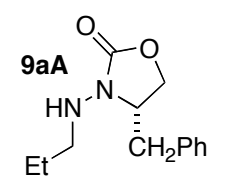

(S)-3-(1'-Propylamino)-4-phenylmethyl-2-oxazolidinone (9aA). Colorless oil; $[\alpha]_{\mathrm{D}}^{23}$ $+42.6^{\circ}$ ( c 0.15, $\mathrm{CHCl}_{3}$ ); IR (film) 3293, 3028, 2961, 1757, 1604, 1497, 1402, $1093 \mathrm{~cm}^{-1}$; ${ }^{1} \mathrm{H}$ NMR $\left(500 \mathrm{MHz}, \mathrm{CDCl}_{3}\right) \delta 7.30$ (dddd, $\left.J=7.0,7.0,1.1,1.1 \mathrm{~Hz}, 2 \mathrm{H}\right), 7.24$ (dddd, $J=$ $7.3,7.3,1.2,1.2 \mathrm{~Hz}, 1 \mathrm{H}), 7.15(\mathrm{ddd}, J=6.8,1.4,1.4 \mathrm{~Hz}, 2 \mathrm{H}), 4.13(\mathrm{dd}, J=8.6,7.7 \mathrm{~Hz}$, $1 \mathrm{H}), 4.02-3.97(\mathrm{~m}, 2 \mathrm{H}), 3.96-3.90(\mathrm{~m}, 1 \mathrm{H}), 3.30(\mathrm{dd}, J=13.6,3.7 \mathrm{~Hz}, 1 \mathrm{H}), 3.00-2.93(\mathrm{~m}, 1 \mathrm{H}), 2.91-2.85$ $(\mathrm{m}, 1 \mathrm{H}), 2.64(\mathrm{dd}, J=13.5,9.7 \mathrm{~Hz}, 1 \mathrm{H}), 1.53(\mathrm{~m}$, apparent sextet, $J=7.3 \mathrm{~Hz}, 2 \mathrm{H}), 0.97(\mathrm{dd}, J=7.4,7.4$ $\mathrm{Hz}, 3 \mathrm{H}) ;{ }^{13} \mathrm{C}$ NMR $\left(125 \mathrm{MHz}, \mathrm{CDCl}_{3}\right) \delta 158.4,135.9,129.0,128.9,127.0,66.2,58.9,52.7,37.7,21.3$, 11.4; MS (CI) $m / z$ (relative intensity) $235\left([\mathrm{M}+\mathrm{H}]^{+}, 100 \%\right.$ ); Anal. Calcd for $\mathrm{C}_{13} \mathrm{H}_{18} \mathrm{~N}_{2} \mathrm{O}_{2}: \mathrm{C}, 66.64 ; \mathrm{H}$, 7.74; N, 11.96. Found: C, 66.39; H, 7.72; N, 11.75.

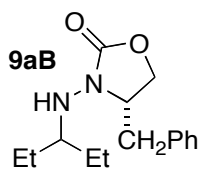

(S)-3-(3'-Pentylamino)-4-phenylmethyl-2-oxazolidinone (9aB). Colorless oil; $[\alpha]_{\mathrm{D}}^{23}$ $+33.5^{\circ}\left(c 0.21, \mathrm{CHCl}_{3}\right.$ ); IR (film) 3291, 3028, 2963, 1758, 1604, 1454, 1239, $1088 \mathrm{~cm}^{-1}$; ${ }^{1} \mathrm{H}$ NMR $\left(500 \mathrm{MHz}, \mathrm{CDCl}_{3}\right.$ ) $\delta 7.32$ (dddd, $\left.J=7.0,7.0,1.6,1.6 \mathrm{~Hz}, 2 \mathrm{H}\right), 7.25$ (dddd, $J=$ $7.3,7.3,1.3,1.3 \mathrm{~Hz}, 1 \mathrm{H}), 7.16(\mathrm{ddd}, J=7.1,1.5,1.5 \mathrm{~Hz}, 2 \mathrm{H}), 4.14(\mathrm{dd}, J=8.8,7.6 \mathrm{~Hz}$, $1 \mathrm{H}), 4.03(\mathrm{dd}, J=8.9,4.8 \mathrm{~Hz}, 1 \mathrm{H}), 3.95(\mathrm{~d}, J=3.8 \mathrm{~Hz}, 1 \mathrm{H}), 3.92-3.87(\mathrm{~m}, 1 \mathrm{H}), 3.34(\mathrm{dd}, J=13.5,3,6$ 
Friestad et al.

$\mathrm{Hz}, 1 \mathrm{H}), 2.98-2.92(\mathrm{~m}, 1 \mathrm{H}), 2.62(\mathrm{dd}, J=13.5,10.0 \mathrm{~Hz}, 1 \mathrm{H}), 1.53-1.45(\mathrm{~m}, 4 \mathrm{H}), 1.00-0.94(\mathrm{~m}, 6 \mathrm{H}) ;{ }^{13} \mathrm{C}$ NMR $\left(125 \mathrm{MHz}, \mathrm{CDCl}_{3}\right) \delta 158.6,136.1,129.1,128.9,127.0,65.7,61.5,59.8,37.0,24.2$, 24.1, 9.7, 9.1; MS (CI) $\mathrm{m} / \mathrm{z}$ (relative intensity) $263\left([\mathrm{M}+\mathrm{H}]^{+}, 100 \%\right.$ ); Anal. Calcd for $\mathrm{C}_{15} \mathrm{H}_{22} \mathrm{~N}_{2} \mathrm{O}_{2}: \mathrm{C}, 68.67 ; \mathrm{H}, 8.45$; N, 10.68. Found: C, 68.44; H, 8.42; N, 10.54 .

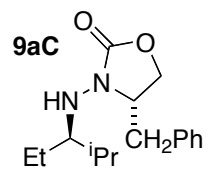

(4S,3'R)-3-(2'-Methyl-3'-pentylamino)-4-phenylmethyl-2-oxazolidinone (9aC). From 3a (32 mg, $0.138 \mathrm{mmol}$ ) and 2-iodopropane by General Procedure $\mathrm{C}$ was obtained 9aC (23 mg, 60\% yield, S,R/S,S = 99:1) as a colorless solid. HPLC retention time 11.3 $\min ; \operatorname{mp~} 75-76{ }^{\circ} \mathrm{C} ;[\alpha]_{\mathrm{D}}{ }^{26}+38.3^{\circ}\left(c 0.65, \mathrm{CHCl}_{3}\right)$; IR (film) 3296, 3089, 3026, 2960, 2927, 1779, 1604, 1491, 1392, 1368, 1252, 1082, 953, $833 \mathrm{~cm}^{-1} ;{ }^{1} \mathrm{H}$ NMR $\left(500 \mathrm{MHz}, \mathrm{CDCl}_{3}\right) \delta 7.29$ (dd, $J=7.2,7.2 \mathrm{~Hz}, 2 \mathrm{H}), 7.23(\mathrm{dd}, J=7.4,7.4 \mathrm{~Hz}, 1 \mathrm{H}), 7.13(\mathrm{~d}, J=7.2 \mathrm{~Hz}, 2 \mathrm{H}), 4.09(\mathrm{dd}, J=8.6,8.6 \mathrm{~Hz}$, $1 \mathrm{H}), 4.00(\mathrm{dd}, J=8.8,4.9 \mathrm{~Hz}, 2 \mathrm{H}), 3.89-3.84(\mathrm{~m}, 1 \mathrm{H}), 3.32(\mathrm{dd}, J=13.4,3.5 \mathrm{~Hz}, 1 \mathrm{H}), 2.75(\mathrm{~m}$, apparent quartet, $J=6.1 \mathrm{~Hz}, 1 \mathrm{H}), 2.59(\mathrm{dd}, J=13.4,10.0 \mathrm{~Hz}, 1 \mathrm{H}), 1.83(\mathrm{~m}$, apparent octet, $J=4.8 \mathrm{~Hz}, 1 \mathrm{H}), 1.52-$ $1.44(\mathrm{~m}, 1 \mathrm{H}), 1.43-1.35(\mathrm{~m}, 1 \mathrm{H}), 0.98-0.92(\mathrm{~m}, 9 \mathrm{H}) ;{ }^{13} \mathrm{C} \mathrm{NMR}\left(125 \mathrm{MHz}, \mathrm{CDCl}_{3}\right) \delta 158.6,136.1,129.1$, $128.9,127.0,65.8,59.7,37.0,28.2,21.5,18.4,17.9,10.2$; MS (CI) $m / z$ (relative intensity) $277\left([\mathrm{M}+\mathrm{H}]^{+}\right.$, $100 \%), 276(\mathrm{M}, 8 \%)$; HRMS (FAB+) calcd for $\mathrm{C}_{16} \mathrm{H}_{24} \mathrm{~N}_{2} \mathrm{O}_{2} \mathrm{Li}: 283.1998$. Found: 283.1993.

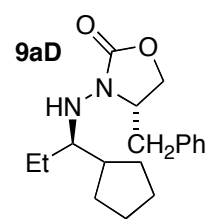

(4S,1'R)-3-(1'-Cyclopentyl-1'-propylamino)-4-phenylmethyl-2-oxazolidinone $[(\boldsymbol{S}, \boldsymbol{R})-9 \mathrm{aD}]$. From 3a $(34 \mathrm{mg}, 0.146 \mathrm{mmol})$ and iodocyclopentane by General Procedure $\mathrm{C}$ was obtained $(\boldsymbol{S}, \boldsymbol{R})-\mathbf{9 a D}(26 \mathrm{mg}, 59 \%$ yield, $\mathrm{S}, \mathrm{R} / \mathrm{S}, \mathrm{S}=96: 4)$ as a colorless oil. HPLC retention time $8.8 \mathrm{~min} ;[\alpha]_{\mathrm{D}}{ }^{26}+17.8^{\circ}\left(c 1.4, \mathrm{CHCl}_{3}\right)$; IR (film) 3306, 3062, 3027, 2953, 2869, 1758, 1604, 1497, 1454, 1399, 1238, 1217, 1092, 1005, $894 \mathrm{~cm}^{-1} ;{ }^{1} \mathrm{H}$ NMR (500 $\left.\mathrm{MHz}, \mathrm{CDCl}_{3}\right) \delta 7.30(\mathrm{dd}, J=7.2,7.2 \mathrm{~Hz}, 2 \mathrm{H}), 7.23(\mathrm{dd}, J=7.4,7.4 \mathrm{~Hz}, 1 \mathrm{H}), 7.14(\mathrm{~d}, J=7.2 \mathrm{~Hz}, 2 \mathrm{H})$, $4.11(\mathrm{dd}, J=8.7,8.7 \mathrm{~Hz}, 1 \mathrm{H}), 4.04-4.00(\mathrm{~m}, 2 \mathrm{H}), 3.87(\mathrm{dddd}, J=10.1,7.3,3.6,3.6 \mathrm{~Hz}, 1 \mathrm{H}), 3.34(\mathrm{dd}, J$ $=13.4,3.3 \mathrm{~Hz}, 1 \mathrm{H}), 2.94-2.90(\mathrm{~m}, 1 \mathrm{H}), 2.58(\mathrm{dd}, J=13.4,10.1 \mathrm{~Hz}, 1 \mathrm{H}), 1.94-1.79(\mathrm{~m}, 2 \mathrm{H}), 1.78-1.72$ $(\mathrm{m}, 1 \mathrm{H}), 1.69-1.41(\mathrm{~m}, 7 \mathrm{H}), 1.28-1.20(\mathrm{~m}, 1 \mathrm{H}), 0.98(\mathrm{dd}, J=7.4,7.4 \mathrm{~Hz}, 3 \mathrm{H}) ;{ }^{13} \mathrm{C} \mathrm{NMR}(125 \mathrm{MHz}$, $\left.\mathrm{CDCl}_{3}\right) \delta 158.2,136.2,129.1,128.9,127.0,65.4,64.8,59.9,40.5,36.7,29.4,25.6,25.4,23.1,8.2 ; \mathrm{MS}$ (CI) $\mathrm{m} / \mathrm{z}$ (relative intensity) $303\left([\mathrm{M}+\mathrm{H}]^{+}, 100 \%\right), 193(100 \%)$; Anal. Calcd for $\mathrm{C}_{18} \mathrm{H}_{26} \mathrm{~N}_{2} \mathrm{O}_{2}: \mathrm{C}, 71.47 ; \mathrm{H}$, 8.67; N, 9.26. Found: C, 71.57; H, 8.84; N, 9.16.

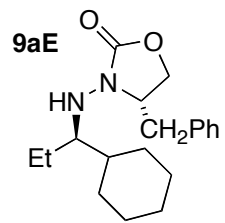

(4S,1'R)-3-(1'-Cyclohexyl-1'-propylamino)-4-phenylmethyl-2-oxazolidinone $[(\boldsymbol{S}, \boldsymbol{R})-\mathbf{9 a E}]$. From 3a $(91 \mathrm{mg}, 0.392 \mathrm{mmol})$ and iodocyclohexane by General Procedure $\mathrm{C}$ was obtained $(\boldsymbol{S}, \boldsymbol{R})-\mathbf{9 a E}(35 \mathrm{mg}, 28 \%$ yield, $\mathrm{S}, \mathrm{R} / \mathrm{S}, \mathrm{S}=97: 3)$ as a colorless oil. HPLC retention time $8.5 \mathrm{~min} ;[\alpha]_{\mathrm{D}}{ }^{26}+38.6^{\circ}\left(c 0.4, \mathrm{CHCl}_{3}\right)$; IR (film) $3299,3027,2926,2852$, 1759, 1604, 1497, 1453, 1398, 1238, 1086, 1029, $893.3 \mathrm{~cm}^{-1} ;{ }^{1} \mathrm{H}$ NMR $(500 \mathrm{MHz}$, $\mathrm{CDCl}_{3}$ ) $\delta 7.32$ (dddd, $\left.J=7.6,7.6,1.3,1.3 \mathrm{~Hz}, 2 \mathrm{H}\right), 7.25$ (dddd, $J=7.4,7.4,1.3,1.3 \mathrm{~Hz}, 1 \mathrm{H}$ ), 7.16 (ddd, $J=7.0,1.3,1.3 \mathrm{~Hz}, 2 \mathrm{H}), 4.13(\mathrm{dd}, J=8.8,7.6 \mathrm{~Hz}, 1 \mathrm{H}), 4.06(\mathrm{~d}, J=4.5 \mathrm{~Hz}, 1 \mathrm{H}), 4.03(\mathrm{dd}, J=8.9,4.9$ Hz, 1H), 3.89 (dddd, $J=13.6,8.3,4.8,3.6 \mathrm{~Hz}, 1 \mathrm{H}), 3.35$ (dd, $J=13.4,3.5 \mathrm{~Hz}, 1 \mathrm{H}), 2.77$ (m, apparent quintet, $J=5.3 \mathrm{~Hz}, 1 \mathrm{H}), 2.62(\mathrm{dd}, J=13.4,10.1 \mathrm{~Hz}, 1 \mathrm{H}), 1.80-1.70(\mathrm{~m}, 5 \mathrm{H}), 1.58-1.44(\mathrm{~m}, 3 \mathrm{H}), 1.28-$ $1.09(\mathrm{~m}, 5 \mathrm{H}), 0.98(\mathrm{dd}, J=7.4,7.4 \mathrm{~Hz}, 3 \mathrm{H}) ;{ }^{13} \mathrm{C} \mathrm{NMR}\left(125 \mathrm{MHz}, \mathrm{CDCl}_{3}\right) \delta$ 158.6, 136.1, 129.1, 128.9, 127.0, 65.7, 65.3 , 59.9, 38.6, 37.0, 29.1, 28.6, 26.7, 26.6, 21.8, 10.1; MS (CI) $\mathrm{m} / \mathrm{z}$ (relative intensity) 317 $\left([\mathrm{M}+\mathrm{H}]^{+}, 73 \%\right), 193(100 \%)$; Anal. Calcd for $\mathrm{C}_{19} \mathrm{H}_{28} \mathrm{~N}_{2} \mathrm{O}_{2}: \mathrm{C}, 72.12 ; \mathrm{H}, 8.92 ; \mathrm{N}, 8.85$. Found: C, 72.16; $\mathrm{H}, 8.83 ; \mathrm{N}, 8.58$.

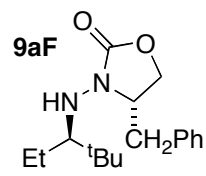

(4S,3'R)-3-(2',2'-Dimethyl-3'-pentylamino)-4-phenylmethyl-2-oxazolidinone $[(S, R)-9 a F]$. From 3a $(35 \mathrm{mg}, 0.150 \mathrm{mmol})$ and 2-iodo-2,2-dimethylpropane by General Procedure $\mathrm{C}$ was obtained $(\boldsymbol{S}, \boldsymbol{R})-\mathbf{9 a F}(24 \mathrm{mg}, 54 \%$ yield, $\mathrm{S}, \mathrm{R} / \mathrm{S}, \mathrm{S}=95: 5)$ as colorless oil. HPLC retention time $7.5 \mathrm{~min} ;[\alpha]_{\mathrm{D}}{ }^{26}+23.4^{\circ}\left(\mathrm{c} 2.6, \mathrm{CHCl}_{3}\right)$; IR (film) 3316, 3087, 3028, 
Friestad et al.

2960, 2872, 1757, 1604, 1498, 1397, 1237, 1198, 1087, 1030, 945, $836 \mathrm{~cm}^{-1} ;{ }^{1} \mathrm{H}$ NMR $(500 \mathrm{MHz}$, $\mathrm{CDCl}_{3}$ ) $\delta 7.32$ (dddd, $\left.J=7.1,7.1,1.4,1.4 \mathrm{~Hz}, 2 \mathrm{H}\right), 7.25$ (dddd, $J=7.4,7.4,1.3,1.3 \mathrm{~Hz}, 1 \mathrm{H}$ ), 7.16 (ddd, $J=7.2,1.4,1.4 \mathrm{~Hz}, 2 \mathrm{H}), 4.15(\mathrm{~d}, J=4.3 \mathrm{~Hz}, 1 \mathrm{H}), 4.11(\mathrm{dd}, J=8.9,7.5 \mathrm{~Hz}, 1 \mathrm{H}), 4.03(\mathrm{dd}, J=8.9,4.1$ $\mathrm{Hz}, 1 \mathrm{H}), 3.93$ (dddd, $J=10.0,6.3,3.7,3.7 \mathrm{~Hz}, 1 \mathrm{H}), 3.36$ (dd, $J=13.5,3.5 \mathrm{~Hz}, 1 \mathrm{H}), 2.71$ (m, apparent quartet, $J=4.7 \mathrm{~Hz}, 1 \mathrm{H}), 2.62(\mathrm{dd}, J=13.5,10.0 \mathrm{~Hz}, 1 \mathrm{H}), 1.73-1.64(\mathrm{~m}, 1 \mathrm{H}), 1.48-1.40(\mathrm{~m}, 1 \mathrm{H}), 1.05$ $(\mathrm{dd}, J=7.6,7.6 \mathrm{~Hz}, 3 \mathrm{H}), 1.00(\mathrm{~s}, 9 \mathrm{H}) ;{ }^{13} \mathrm{C} \mathrm{NMR}\left(125 \mathrm{MHz}, \mathrm{CDCl}_{3}\right) \delta 158.3,136.1,129.1,128.8,126.9$, 69.6, 65.4, 60.3, 36.7, 34.2, 26.8, 23.0, 13.6; MS (CI) $\mathrm{m} / \mathrm{z}$ (relative intensity) $291\left([\mathrm{M}+\mathrm{H}]^{+}, 53 \%\right), 193$ (100\%); Anal. Calcd for $\mathrm{C}_{17} \mathrm{H}_{26} \mathrm{~N}_{2} \mathrm{O}_{2}$ : C, 70.31; H, 9.02; N, 9.65. Found: C, 70.17; H, 9.01; N, 9.65.

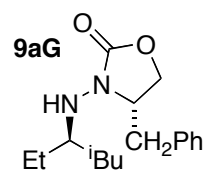

(4S,3'R)-3-(5'-Methyl-3'-hexanamino)-4-phenylmethyl-2-oxazolidinone (9aG). From 3a $(50.5 \mathrm{mg}, 0.218 \mathrm{mmol})$ and isobutyl iodide $(0.25 \mathrm{~mL}, 2.2 \mathrm{mmol})$ by General Procedure $\mathrm{C}$ was obtained $9 \mathbf{a B}(6.7 \mathrm{mg}, 12 \%$ yield) and $\mathbf{9 a G}(5.7 \mathrm{mg}, 6 \%$ yield) as a colorless oil; $[\alpha]_{\mathrm{D}}{ }^{25}+19.5^{\circ}\left(c 0.85, \mathrm{CHCl}_{3}\right.$ ); IR (film) 3299, 3028, 2958, 2872, 1758, 1497, 1454, 1399, 1238, 1091, 1030, 743, $702 \mathrm{~cm}^{-1} ;{ }^{1} \mathrm{H}$ NMR $\left(500 \mathrm{MHz}, \mathrm{CDCl}_{3}\right) \delta 7.32(\mathrm{dd}, J=7.6,7.6$ $\mathrm{Hz}, 2 \mathrm{H}), 7.25(\mathrm{dd}, J=7.4,7.4 \mathrm{~Hz}, 1 \mathrm{H}), 7.16(\mathrm{~d}, J=7.0 \mathrm{~Hz}, 2 \mathrm{H}), 4.14(\mathrm{dd}, J=8.2,8.2 \mathrm{~Hz}, 1 \mathrm{H}), 4.03$ $(\mathrm{dd}, J=8.9,4.4 \mathrm{~Hz}, 1 \mathrm{H}), 3.92-3.87(\mathrm{~m}, 1 \mathrm{H}), 3.97($ br s, $1 \mathrm{H}), 3.35(\mathrm{dd}, J=13.5,3.4 \mathrm{~Hz}, 1 \mathrm{H}), 3.12-3.03$ $(\mathrm{m}, 1 \mathrm{H}), 2.60(\mathrm{dd}, J=13.4,10.1 \mathrm{~Hz}, 1 \mathrm{H}), 1.82-1.73(\mathrm{~m}, 1 \mathrm{H}), 1.58-1.42(\mathrm{~m}, 2 \mathrm{H}), 1.29(\mathrm{dd}, J=6.9,6.9$ $\mathrm{Hz}, 2 \mathrm{H}), 0.99-0.94(\mathrm{~m}, 9 \mathrm{H}) ;{ }^{13} \mathrm{C} \mathrm{NMR}\left(125 \mathrm{MHz}, \mathrm{CDCl}_{3}\right) \delta 158.5,136.1,129.1,128.9,127.0,65.6$, $60.1,58.4,41.1,36.8,25.4,24.9,23.1,22.7,8.9 ; \mathrm{MS}(\mathrm{CI}) \mathrm{m} / \mathrm{z}$ (relative intensity) $291\left([\mathrm{M}+\mathrm{H}]^{+}, 100 \%\right)$; Anal. Calcd for $\mathrm{C}_{17} \mathrm{H}_{26} \mathrm{~N}_{2} \mathrm{O}_{2}$ : C, 70.31; H, 9.02; N, 9.65. Found: C, 70.21; H, 8.97; N, 9.55.

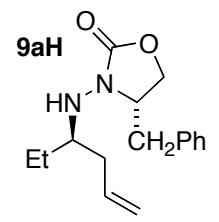

(4S,1'R)-3-(1'-Ethyl-3'-butenylamino)-4-phenylmethyl-2-oxazolidinone (9aH). From 3a $(66.6 \mathrm{mg}, 0.287 \mathrm{mmol})$ and allyl iodide $(0.26 \mathrm{~mL}, 2.8 \mathrm{mmol})$ by General Procedure $\mathrm{C}$ was obtained $9 \mathbf{a H}(5.7 \mathrm{mg}$, $7 \%$ yield) as a colorless oil. This material was identified by comparison with a known sample. ${ }^{5}$

Tin-Free Radical Addition Using $\mathrm{InCl}_{3}$ with Triethylborane Initiation (Table 5): General Procedure D. The standard method for tin-mediated radical addition (General Procedure C) was employed, with the following modifications: $\mathrm{InCl}_{3}$ was substituted for $\mathrm{ZnCl}_{2}$, and $\mathrm{Bu}_{3} \mathrm{SnH}$ was omitted.

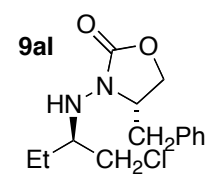

(4S,2'R)-3-(1'-Chloro-2'-butanamino)-4-phenylmethyl-2-oxazolidinone (9aI). From 3a (48 mg, $0.21 \mathrm{mmol})$ and chloroiodomethane $(0.15 \mathrm{ml}, 2.07 \mathrm{mmol})$ by General Procedure D was obtained 9aI (19 mg, 33\% yield) as colorless oil. $[\alpha]_{\mathrm{D}}^{27}+44.9^{\circ}(c 3.4$, $\mathrm{CHCl}_{3}$ ); IR (film) 3286, 3028, 2966, 2878, 1758, 1497, 1400, 1240, 1217, 1091, 1207, 745, $702 \mathrm{~cm}^{-1} ;{ }^{1} \mathrm{H} \mathrm{NMR}\left(500 \mathrm{MHz}, \mathrm{CDCl}_{3}\right) \delta 7.31(\mathrm{dd}, J=8.0,8.0 \mathrm{~Hz}, 2 \mathrm{H}), 7.25(\mathrm{dd}, J=7.9,7.9 \mathrm{~Hz}$, $1 \mathrm{H}), 7.16(\mathrm{~d}, J=7.6 \mathrm{~Hz}, 2 \mathrm{H}), 4.13(\mathrm{dd}, J=8.6,8.6 \mathrm{~Hz}, 1 \mathrm{H}), 4.03(\mathrm{dd}, J=8.9,5.8 \mathrm{~Hz}, 1 \mathrm{H}), 3.93-3.88$ $(\mathrm{m}, 1 \mathrm{H}), 4.25-3.85(\mathrm{br} \mathrm{s}, 1 \mathrm{H}), 3.62\left(\mathrm{ABX}, \Delta v_{\mathrm{AB}}=28 \mathrm{~Hz}, J_{\mathrm{AB}}=11.4 \mathrm{~Hz}, J_{\mathrm{AX}}=4.6 \mathrm{~Hz}, J_{\mathrm{BX}}=4.9 \mathrm{~Hz}, 2 \mathrm{H}\right)$, $3.41(\mathrm{dd}, J=13.4,3.6 \mathrm{~Hz}, 1 \mathrm{H}), 3.24$ (dddd, $J=5.5,5.5,5.5,5.5 \mathrm{~Hz}, 1 \mathrm{H}), 2.63(\mathrm{dd}, J=13.3,10.1 \mathrm{~Hz}$, $1 \mathrm{H}), 1.70-1.56(\mathrm{~m}, 2 \mathrm{H}), 1.01(\mathrm{t}, J=7.4 \mathrm{~Hz}, 3 \mathrm{H}) ;{ }^{13} \mathrm{C} \mathrm{NMR}\left(125 \mathrm{MHz}, \mathrm{CDCl}_{3}\right) \delta 159.0,135.8,129.0$, 128.9, 127.1, 66.2, 61.3, 59.7, 45.5, 37.1, 23.4, 9.8; MS (CI) $\mathrm{m} / z$ (relative intensity) $283\left([\mathrm{M}+\mathrm{H}]^{+}\right.$, $100 \%$ ); Anal. Calcd for $\mathrm{C}_{14} \mathrm{H}_{19} \mathrm{~N}_{2} \mathrm{O}_{2} \mathrm{Cl}$ : C, 59.47; H, 6.77; N, 9.91. Found: C, 59.69; H, 6.82; N, 9.80.

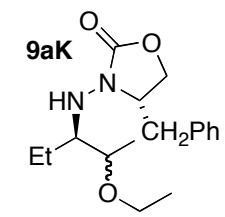

Photolytic Tin-Mediated Radical Addition (Table 6): Hydrazines 9aB and 9aK (General Procedure E). To a solution of hydrazone $3 \mathbf{a}(60 \mathrm{mg}, 0.26 \mathrm{mmol})$ and $\mathrm{InCl}_{3}$ (132 mg, $0.60 \mathrm{mmol})$ in $\mathrm{CH}_{2} \mathrm{Cl}_{2} / \mathrm{Et}_{2} \mathrm{O}(2 \mathrm{~mL} / 2 \mathrm{~mL})$ was added EtI $(41 \mu \mathrm{L}, 0.52 \mathrm{mmol})$, $\left(\mathrm{CH}_{3}\right)_{3} \mathrm{SnSn}\left(\mathrm{CH}_{3}\right)_{3}(102 \mathrm{mg}, 0.31 \mathrm{mmol})$ and acetone $(95 \mu \mathrm{L}, 1.30 \mathrm{mmol})$ and the mixture was irradiated for $18 \mathrm{~h}$ under $\mathrm{N}_{2}$. Stannanes were removed by dilution with EtOAc, stirring overnight with excess KF, and filtration through a short pad of silica gel. Concentration 
and gradient flash chromatography (hexane to hexane/EtOAc 3:1) afforded 9aB (17 mg, 25\% yield) and 9aK (7 mg major isomer, $4 \mathrm{mg}$ minor isomer, $14 \%$ yield). (4S,3' $\boldsymbol{R})$-3-(2'-Ethoxy-3'-pentanamino)-4phenylmethyl-2-oxazolidinone 9aK (major diastereomer): Colorless oil; $[\alpha]_{\mathrm{D}}^{23}+20.0^{\circ}(c) 0.35$, $\mathrm{CHCl}_{3}$ ); IR (film) 3283, 3028, 2972, 2934, 2876, 1757, 1498, 1454, 1402, 1381, 1288, 1238, 1216, 1090, 1004, 746, $701 \mathrm{~cm}^{-1}$; ${ }^{1} \mathrm{H}$ NMR $\left(500 \mathrm{MHz} \mathrm{CDCl}_{3}\right) \delta 7.38-7.12(\mathrm{~m}, 5 \mathrm{H}), 4.14(\mathrm{dd}, J=8.7,8.7 \mathrm{~Hz}$, $1 \mathrm{H}), 4.04(\mathrm{dd}, J=9.4,3.8 \mathrm{~Hz}, 1 \mathrm{H}), 3.99-3.92(\mathrm{~m}, 1 \mathrm{H}), 3.69-3.60(\mathrm{~m}, 2 \mathrm{H}), 3.58-3.49(\mathrm{~m}, 1 \mathrm{H}), 3.37(\mathrm{dd}, J$ $=13.9,4.5 \mathrm{~Hz}, 1 \mathrm{H}), 3.13(\mathrm{ddd}, J=6.8,6.8,3.4 \mathrm{~Hz}, 1 \mathrm{H}), 2.63(\mathrm{dd}, J=13.6,9.8 \mathrm{~Hz}, 1 \mathrm{H}), 1.64-1.52(\mathrm{~m}$, $1 \mathrm{H}), 1.52-1.39(\mathrm{~m}, 1 \mathrm{H}), 1.23(\mathrm{dd}, J=7.2,7.2 \mathrm{~Hz}, 3 \mathrm{H}), 1.17(\mathrm{~d}, J=6.4 \mathrm{~Hz}, 3 \mathrm{H}), 0.98(\mathrm{dd}, J=7.2,7.2$ $\mathrm{Hz}, 3 \mathrm{H}) ;{ }^{13} \mathrm{C} \mathrm{NMR}\left(125 \mathrm{MHz}, \mathrm{CDCl}_{3}\right) \delta 158.0,136.2,129.2,128.4,126.9,74.7,65.4,64.1,62.3,59.6$, 36.6, 21.8, 15.6, 14.3, 10.6; MS (CI) $\mathrm{m} / \mathrm{z}$ (relative intensity) $307\left([\mathrm{M}+\mathrm{H}]^{+}, 100 \%\right)$; HRMS (EI) calcd for $\mathrm{C}_{17} \mathrm{H}_{26} \mathrm{~N}_{2} \mathrm{O}_{3}$ : 306.1943. Found: 306.1942. 9aK (minor diastereomer). Colorless oil; $[\alpha]_{\mathrm{D}}^{23}+4.2^{\circ}(c$ 0.80, $\mathrm{CHCl}_{3}$ ); IR (film) 3299, 3028, 2972, 2931, 1760, 1455, 1400, 1288, 1238, 1100, 1030, 743, 702 $\mathrm{cm}^{-1} ;{ }^{1} \mathrm{H}$ NMR $\left(500 \mathrm{MHz}, \mathrm{CDCl}_{3}\right) \delta$ 7.39-7.12 (m, 5H), $4.13(\mathrm{dd}, J=8.7,8.7 \mathrm{~Hz}, 1 \mathrm{H}), 4.03(\mathrm{dd}, J=9.1$, $4.5 \mathrm{~Hz}, 1 \mathrm{H}), 3.97-3.89(\mathrm{~m}, 1 \mathrm{H}), 3.71-3.61(\mathrm{~m}, 1 \mathrm{H}), 3.49(\mathrm{~m}$, apparent quintet, $J=6.0 \mathrm{~Hz}, 1 \mathrm{H}), 3.44-3.32$ $(\mathrm{m}, 2 \mathrm{H}), 3.05(\mathrm{ddd}, J=6.4,4.9,4.9 \mathrm{~Hz}, 1 \mathrm{H}), 2.62(\mathrm{dd}, J=13.6,10.2 \mathrm{~Hz}, 1 \mathrm{H}), 1.74-1.60(\mathrm{~m}, 1 \mathrm{H}), 1.53-$ $1.41(\mathrm{~m}, 1 \mathrm{H}), 1.30-1.17(\mathrm{~m}, 6 \mathrm{H}), 0.98(\mathrm{dd}, J=7.2,7.2 \mathrm{~Hz}, 3 \mathrm{H}) ;{ }^{13} \mathrm{C} \mathrm{NMR}\left(125 \mathrm{MHz}, \mathrm{CDCl}_{3}\right) \delta 158.2$, 136.1, 129.1, 128.9, 127.0, 74.6, 65.5, 63.7, 63.4, 59.7, 36.9, 21.0, 15.6, 15.2, 8.6; MS (CI) $\mathrm{m} / z$ (relative intensity) $307\left([\mathrm{M}+\mathrm{H}]^{+}, 100 \%\right)$; HRMS (EI) calcd for $\mathrm{C}_{17} \mathrm{H}_{26} \mathrm{~N}_{2} \mathrm{O}_{3}: 306.1943$. Found: 306.1942 .

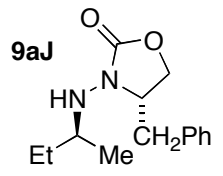

(4S,2'S)-3-(2'-Butanamino)-4-phenylmethyl-2-oxazolidin-one (9aJ). From 3a (60 $\mathrm{mg}, 0.26 \mathrm{mmol})$ and iodomethane $(161 \mu \mathrm{L}, 2.6 \mathrm{mmol})$ by General Procedure E 9aJ (6 $\mathrm{mg}, 9 \%$ yield) as colorless oil. $[\alpha]_{\mathrm{D}}{ }^{26}+49.2^{\circ}\left(c 0.65, \mathrm{CHCl}_{3}\right)$; IR (film) $3288,3028,2966$, 2877, 1758, 1498, 1454, 1399, 1218, 1088, 745, $703 \mathrm{~cm}^{-1} ;{ }^{1} \mathrm{H}$ NMR $\left(500 \mathrm{MHz}, \mathrm{CDCl}_{3}\right) \delta$ $7.32(\mathrm{dd}, J=7.1,7.1 \mathrm{~Hz}, 2 \mathrm{H}), 7.25(\mathrm{dd}, J=7.2,7.2 \mathrm{~Hz}, 1 \mathrm{H}), 7.16(\mathrm{~d}, J=7.0 \mathrm{~Hz}, 2 \mathrm{H}), 4.15(\mathrm{dd}, J=8.3$, $8.3 \mathrm{~Hz}, 1 \mathrm{H}), 4.03(\mathrm{dd}, J=8.8,5.3 \mathrm{~Hz}, 1 \mathrm{H}), 3.93-3.88(\mathrm{~m}, 1 \mathrm{H}), 4.30-3.70$ (br s, $1 \mathrm{H}), 3.33(\mathrm{dd}, J=13.5$, $3.6 \mathrm{~Hz}, 1 \mathrm{H}), 3.19-3.10(\mathrm{~m}, 1 \mathrm{H}), 2.62(\mathrm{dd}, J=13.4,9.9 \mathrm{~Hz}, 1 \mathrm{H}), 1.60-1.57(\mathrm{~m}, 1 \mathrm{H}), 1.41-1.32(\mathrm{~m}, 1 \mathrm{H})$, $1.08(\mathrm{~d}, J=6.4 \mathrm{~Hz}, 3 \mathrm{H}), 0.95(\mathrm{dd}, J=7.5 \mathrm{~Hz}, 3 \mathrm{H}) ;{ }^{13} \mathrm{C} \mathrm{NMR}\left(125 \mathrm{MHz}, \mathrm{CDCl}_{3}\right) \delta$ 158.8, 136.0, 129.1, 128.8, 127.0, 66.0, 59.7, 56.0, 37.3, 27.5, 18.0, 9.7; MS (CI) m/z (relative intensity) $249\left([\mathrm{M}+\mathrm{H}]^{+}\right.$, $100 \%$ ); Anal. Calcd for $\mathrm{C}_{14} \mathrm{H}_{20} \mathrm{~N}_{2} \mathrm{O}_{2}:$ C, 67.71; H, 8.12; N, 11.28. Found: C, 67.79; H, 8.09; N, 11.22.

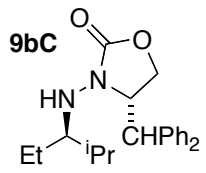

(4S,3'R)-3-(2'-Methyl-3'-pentylamino)-4-diphenylmethyl-2-oxazolidinone (9bC). From $3 \mathbf{b}$ (29 mg, $0.094 \mathrm{mmol}$ ) and 2-iodopropane by General Procedure $\mathrm{C}$ was obtained 9bC (5 mg, 15\% yield, S,R/S,S >98:2, minor diastereomer not detected by ${ }^{1} \mathrm{H} \mathrm{NMR}$ ) as a colorless crystalline solid. mp $114-115^{\circ} \mathrm{C} ;[\alpha]_{\mathrm{D}}{ }^{23}+51.1^{\circ}\left(c 0.7, \mathrm{CHCl}_{3}\right)$; IR (film) 3316 , 3029, 2961, 1756, 1495, 1454, 1405, 1219, $1091 \mathrm{~cm}^{-1} ;{ }^{1} \mathrm{H}$ NMR $\left(500 \mathrm{MHz}, \mathrm{CDCl}_{3}\right) \delta 7.33-7.14(\mathrm{~m}$, $10 \mathrm{H}), 4.58(\mathrm{~d}, J=5.8 \mathrm{~Hz}, 1 \mathrm{H}), 4.57-4.54(\mathrm{~m}, 1 \mathrm{H}), 4.37(\mathrm{dd}, J=9.0,8.2 \mathrm{~Hz}, 1 \mathrm{H}), 4.22(\mathrm{dd}, J=9.1,3.4$ $\mathrm{Hz}, 1 \mathrm{H}), 3.61(\mathrm{~d}, J=3.3 \mathrm{~Hz}, 1 \mathrm{H}), 2.74-2.70(\mathrm{~m}, 1 \mathrm{H}), 1.77-1.70(\mathrm{~m}, 1 \mathrm{H}), 1.34-1.27(\mathrm{~m}, 1 \mathrm{H}), 1.24-1.11$ $(\mathrm{m}, 1 \mathrm{H}), 0.81(\mathrm{~d}, J=6.9 \mathrm{~Hz}, 3 \mathrm{H}), 0.78(\mathrm{~d}, J=6.9 \mathrm{~Hz}, 3 \mathrm{H}), 0.72(\mathrm{t}, J=7.5 \mathrm{~Hz}, 3 \mathrm{H}) ;{ }^{13} \mathrm{C}$ NMR $(125$ $\left.\mathrm{MHz}, \mathrm{CDCl}_{3}\right) \delta 157.0,140.8,139.2,128.9$ (2C), 128.7, 128.3, 127.4, 127.0, 64.4, 64.2, 59.4, 51.5, 28.1, 21.4, 18.0, 17.7, 10.3; MS (CI) $\mathrm{m} / \mathrm{z}$ (relative intensity) $353.7\left([\mathrm{M}+\mathrm{H}]^{+}, 100 \%\right)$; Anal. Calcd for $\mathrm{C}_{22} \mathrm{H}_{28} \mathrm{~N}_{2} \mathrm{O}_{2}: \mathrm{C}, 74.97 ; \mathrm{H}, 8.01 ; \mathrm{N}, 7.95$. Found: C, 74.85; H, 8.06; N, 7.74.

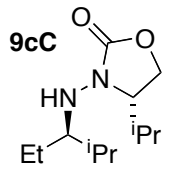

(4S,3'R)-3-(2'-Methyl-3'-pentylamino)-4-isopropyl-2-oxazolidinone (9cC). From 3c $(147 \mathrm{mg}, 0.80 \mathrm{mmol})$ and 2-iodopropane by General Procedure C was obtained 9cC (28 mg, $16 \%$ yield as a colorless oil. IR (film) 3300, 2962, 1757, 1465, 1399, 1220, $1090 \mathrm{~cm}^{-1} ;{ }^{1} \mathrm{H}$ NMR $\left(500 \mathrm{MHz}, \mathrm{CDCl}_{3}\right) \delta 4.21(\mathrm{dd}, J=8.8,8.7 \mathrm{~Hz}, 1 \mathrm{H}), 4.05(\mathrm{dd}, J=9.0,4.2 \mathrm{~Hz}, 1 \mathrm{H})$, 3.89 (br s, $1 \mathrm{H}), 3.69-3.66(\mathrm{~m}, 1 \mathrm{H}), 2.76-2.73(\mathrm{~m}$, apparent q, $J=5.2 \mathrm{~Hz}, 1 \mathrm{H}), 2.30-2.22(\mathrm{~m}, 1 \mathrm{H}), 1.82-$ $1.76(\mathrm{~m}, 1 \mathrm{H}), 1.49-1.36(\mathrm{~m}, 2 \mathrm{H}), 0.97-0.88(\mathrm{~m}, 12 \mathrm{H}), 0.85(\mathrm{~d}, J=6.9 \mathrm{~Hz}, 3 \mathrm{H}) ;{ }^{13} \mathrm{C} \mathrm{NMR}(125 \mathrm{MHz}$, 
Friestad et al.

$\left.\mathrm{CDCl}_{3}\right) \delta 158.6,65.1,62.3,61.8,28.2,27.4,21.6,18.1,18.0,17.7,14.9,10.1 ; \mathrm{MS}$ (CI) $\mathrm{m} / z$ (relative intensity) $229.2\left([\mathrm{M}+\mathrm{H}]^{+}, 100 \%\right)$; Anal. Calcd for $\mathrm{C}_{12} \mathrm{H}_{24} \mathrm{~N}_{2} \mathrm{O}_{2}: \mathrm{C}, 63.12 ; \mathrm{H}, 10.59 ; \mathrm{N}, 12.27$. Found: C, 63.09; H, 10.69; N, 12.11. Diastereomers of $9 \mathbf{c C}$ were not resolved by ${ }^{1} \mathrm{H}$ NMR or any of the chromatographic methods attempted, including several chiral HPLC columns. Thus the diastereomer ratio was not available.

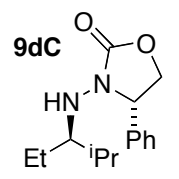

(4S,3'R)-3-(2'-Methyl-3'-pentylamino)-4-phenyl-2-oxazolidinone (9dC). From 3d (83 $\mathrm{mg}, 0.40 \mathrm{mmol}$ ) and 2-iodopropane by General Procedure $\mathrm{C}$ was obtained 9dC (35 mg $35 \%$ yield, $\mathrm{S}, \mathrm{R} / \mathrm{S}, \mathrm{S}=94: 6)$ as a colorless oil. $[\alpha]_{\mathrm{D}}{ }^{23}+32.0^{\circ}\left(c 0.3, \mathrm{CHCl}_{3}\right)$; IR (film) 3304, 2922, 1761, 1459, 1391, 1240, 1086, $1039 \mathrm{~cm}^{-1} ;{ }^{1} \mathrm{H}$ NMR $\left(500 \mathrm{MHz}, \mathrm{CDCl}_{3}\right) \delta$ 7.44-7.33 $(\mathrm{m}, 5 \mathrm{H}), 4.81(\mathrm{dd}, J=8.4,6.4 \mathrm{~Hz}, 1 \mathrm{H}), 4.61(\mathrm{dd}, J=8.8,8.7 \mathrm{~Hz}, 1 \mathrm{H}), 4.24(\mathrm{dd}, J=8.8,6.4 \mathrm{~Hz}, 1 \mathrm{H})$, 2.54-2.51 (m, 1H), 1.67-1.62 (m, 1H), 1.44-1.39 (m, 1H), 1.38-1.27 (m, 2H), 0.83 (d, J = 6.9 Hz, 3H), $0.77(\mathrm{~d}, J=6.8 \mathrm{~Hz}, 3 \mathrm{H}), 0.71(\mathrm{t}, J=7.3 \mathrm{~Hz}, 3 \mathrm{H}) ;{ }^{13} \mathrm{C} \mathrm{NMR}\left(125 \mathrm{MHz}, \mathrm{CDCl}_{3}\right) \delta 158.3,137.2,129.1$ (2C), 127.5, 68.5, 64.4, 61.4, 28.3, 20.9, 18.2, 17.2, 10.2; MS (CI) $\mathrm{m} / \mathrm{z}$ (relative intensity) 263.1 $\left([\mathrm{M}+\mathrm{H}]^{+}, 100 \%\right), 219.0(20 \%)$; Anal. Calcd for $\mathrm{C}_{15} \mathrm{H}_{22} \mathrm{~N}_{2} \mathrm{O}_{2}: \mathrm{C}, 68.67 ; \mathrm{H}, 8.45 ; \mathrm{N}, 10.68$. Found: C, $68.89 ; \mathrm{H}, 8.43 ; \mathrm{N}, 10.51$.

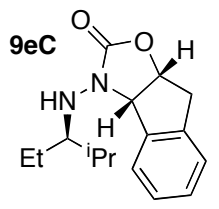

(4S,3'R)-3-(2'-Methyl-3'-pentylamino)-4-phenyl-2-oxazolidinone (9eC). From 3e $(131 \mathrm{mg}, 0.57 \mathrm{mmol})$ and 2-iodopropane by General Procedure $\mathrm{C}$ was obtained $9 \mathrm{eC}(80$ $\mathrm{mg}, 50 \%$ yield, $\mathrm{S}, \mathrm{R} / \mathrm{S}, \mathrm{S}=95: 5)$ as a colorless oil. $[\alpha]_{\mathrm{D}}{ }^{23}-28.9^{\circ}\left(c 0.4, \mathrm{CHCl}_{3}\right)$; IR (film) $3304,3031,2961,1753,1462,1381,1201,1106,1035 \mathrm{~cm}^{-1} ;{ }^{1} \mathrm{H}$ NMR $\left(500 \mathrm{MHz}, \mathrm{CDCl}_{3}\right)$ $\delta 7.67(\mathrm{~d}, J=7.5 \mathrm{~Hz}, 1 \mathrm{H}), 7.35-7.25(\mathrm{~m}, 3 \mathrm{H}), 5.25-5.21(\mathrm{~m}, 1 \mathrm{H}), 5.09(\mathrm{~d}, J=6.9 \mathrm{~Hz}, 1 \mathrm{H}), 4.02($ br s, $1 \mathrm{H}), 3.33\left(\mathrm{ABX}, \Delta v_{\mathrm{AB}}=32.2 \mathrm{~Hz}, J_{\mathrm{AB}}=17.5 \mathrm{~Hz}, J_{\mathrm{AX}}=6.1 \mathrm{~Hz}, J_{\mathrm{BX}}=0 \mathrm{~Hz}, 2 \mathrm{H}\right), 2.94-2.91(\mathrm{~m}, 1 \mathrm{H}), 1.88-$ $1.82(\mathrm{~m}, 1 \mathrm{H}), 1.63-1.50(\mathrm{~m}, 2 \mathrm{H}), 0.96(\mathrm{t}, J=7.4 \mathrm{~Hz}, 3 \mathrm{H}), 0.91(\mathrm{~d}, J=6.9 \mathrm{~Hz}, 6 \mathrm{H}) ;{ }^{13} \mathrm{C} \mathrm{NMR}(125 \mathrm{MHz}$, $\left.\mathrm{CDCl}_{3}\right) \delta 157.5,140.2,138.3,129.5,127.2,126.1,125.3,76.5,65.6,64.9,38.9,28.2,21.7,18.2,18.1$, 10.1; MS (CI) $\mathrm{m} / \mathrm{z}$ (relative intensity) 275.4 ([M+H] $]^{+}, 100 \%$ ); Anal. Calcd for $\mathrm{C}_{16} \mathrm{H}_{22} \mathrm{~N}_{2} \mathrm{O}_{2}: \mathrm{C}, 70.04 ; \mathrm{H}$, 8.08; N, 10.21. Found: C, 69.85; H, 8.10; N, 10.08 .

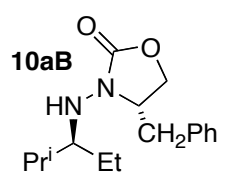

(4S,3'S)-3-(2'-Methyl-3'-pentylamino)-4-phenylmethyl-2-oxazolidinone (10aB). From $4(86 \mathrm{mg}, 0.35 \mathrm{mmol})$ and ethyl iodide $(0.28 \mathrm{~mL}, 3.5 \mathrm{mmol})$ by General Procedure $\mathrm{C}$ was obtained unreacted $4(75.4 \mathrm{mg}, 88 \%)$ and ethyl adduct 10aB (5.8 $\mathrm{mg}, 6 \%$ yield) as colorless oil. This material was identified by comparison with a known sample. ${ }^{6}$

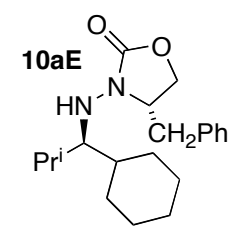

(4S,1'R)-3-(1'-Cyclohexyl-2'-methyl-1'-propylamino)-4-phenylmethyl-2oxazolidinone (10aE). From $4(40 \mathrm{mg}, 0.16 \mathrm{mmol})$ and cyclohexyl iodide $(0.21 \mathrm{~mL}$, $1.60 \mathrm{mmol})$ by General Procedure C was obtained unreacted 4 (31 $\mathrm{mg}, 77 \%)$ and cyclohexyl adduct $\mathbf{1 0 a E}(5 \mathrm{mg}, 9 \%$ yield $)$ as colorless oil. $[\alpha]_{\mathrm{D}}{ }^{23}+11.35^{\circ}\left(c 0.37, \mathrm{CHCl}_{3}\right)$; ${ }^{1} \mathrm{H}$ NMR $\left(500 \mathrm{MHz} \mathrm{CDCl}_{3}\right) \delta$ 7.34-7.13 (m, 5H), 4.09-4.02 (m, 3H), 3.94-3.90 (m, 1H), $3.34(\mathrm{dd}, J=$ 13.4, 3.4 Hz, 1H), 2.63-2.56 (m, 2H), 1.95-1.88 (m, 1H), 1.82-1.74 (m, 3H), 1.68-1.65 (m, 1H), 1.48$1.44(\mathrm{~m}, 1 \mathrm{H}), 1.34-1.14(\mathrm{~m}, 6 \mathrm{H}), 1.04(\mathrm{~d}, J=6.9 \mathrm{~Hz}, 3 \mathrm{H}), 0.97(\mathrm{~d}, J=6.9 \mathrm{~Hz}, 3 \mathrm{H}) ;{ }^{13} \mathrm{C} \mathrm{NMR}(500 \mathrm{MHz}$, $\left.\mathrm{CDCl}_{3}\right) \delta 158.3,136.2,129.1,128.9,129.0,69.1,65.4,59.6,39.1,36.6,31.1,29.7,28.8,27.0,26.8$, 26.6, 20.2, 19.0.

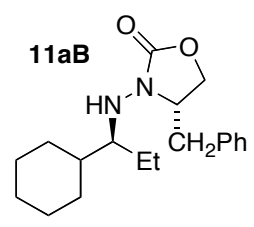

(4S,1'S)-3-(1'-Cyclohexyl-1'-propylamino)-4-phenylmethyl-2-oxazolidinone (11aB). From $5(60 \mathrm{mg}, 0.21 \mathrm{mmol})$ and ethyl iodide $(0.17 \mathrm{~mL}, 2.1 \mathrm{mmol})$ by General Procedure C was obtained unreacted $5(37 \mathrm{mg}, 61 \%)$ and ethyl adduct 11aB (10 mg, 15\% yield) as colorless oil. This material was identified by comparison with a known sample. ${ }^{6}$ 


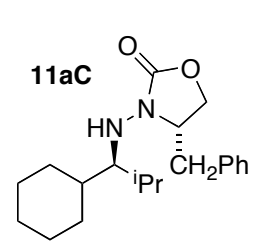

(4S,1'S)-3-(1'-Cyclohexyl-2'-methyl-1'-propylamino)-4-phenylmethyl-2oxazolidinone (11aC). From $5(58 \mathrm{mg}, 0.20 \mathrm{mmol})$ and isopropyl iodide $(0.20 \mathrm{~mL}$, $2.0 \mathrm{mmol}$ ) by General Procedure $\mathrm{C}$ was obtained unreacted 5 (46 $\mathrm{mg}, 79 \%)$ and cyclohexyl adduct $11 \mathrm{aC}\left(6 \mathrm{mg}, 9 \%\right.$ yield) as colorless oil. $[\alpha]_{\mathrm{D}}{ }^{20}+16.6^{\circ}(c 0.53$, $\left.\mathrm{CHCl}_{3}\right) ;{ }^{1} \mathrm{H}$ NMR $\left(500 \mathrm{MHz}, \mathrm{CDCl}_{3}\right) \delta 7.34-7.13(\mathrm{~m}, 5 \mathrm{H}), 4.08(\mathrm{dd}, J=8.8,7.4 \mathrm{~Hz}$, $1 \mathrm{H}), 4.02-4.00(\mathrm{~m}, 2 \mathrm{H}), 3.92-3.86(\mathrm{~m}, 1 \mathrm{H}), 3.35(\mathrm{dd}, J=13.4,3.4 \mathrm{~Hz}, 1 \mathrm{H}), 2.61-2.56$ $(\mathrm{m}, 2 \mathrm{H}), 1.94-1.87(\mathrm{~m}, 1 \mathrm{H}), 1.80-1.65(\mathrm{~m}, 4 \mathrm{H}), 1.52-1.48(\mathrm{~m}, 1 \mathrm{H}), 1.27-1.13(\mathrm{~m}, 6 \mathrm{H}), 1.04(\mathrm{~d}, J=7.0$ $\mathrm{Hz}, 3 \mathrm{H}), 0.99$ (d, $J=7.0 \mathrm{~Hz}, 3 \mathrm{H}) ;{ }^{13} \mathrm{C} \mathrm{NMR}\left(500 \mathrm{MHz}, \mathrm{CDCl}_{3}\right) \delta 158.3,136.2,129.1,128.9,127.0$, $69.1,65.4,59.5,39.7,36.7,30.7,29.8,28.5,27.1,26.9,26.8,20.7,18.9$.

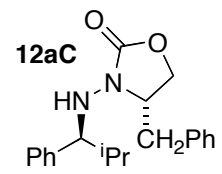

(4S,1'S)-3-(2'-Methyl-1'-phenyl-1'-propylamino)-4-phenylmethyl-2oxazolidinone $[(S, S)-12 a C]$. From $7(47 \mathrm{mg}, 0.168 \mathrm{mmol})$ and 2-iodopropane by General Procedure $\mathrm{C}$ was obtained $(\boldsymbol{S}, \boldsymbol{S}) \mathbf{- 1 2 a C}(23 \mathrm{mg}, 42 \%$ yield, $\mathrm{S}, \mathrm{S} / \mathrm{S}, \mathrm{R}=99: 1)$ as a colorless solid. GCMS retention time $6.2 \mathrm{~min} ; \mathrm{mp} 91-93{ }^{\circ} \mathrm{C} ;[\alpha]_{\mathrm{D}}{ }^{26}-18.2^{\circ}(c 0.8$, $\mathrm{CHCl}_{3}$ ); IR (film) 3309, 3062, 3028, 2960, 2873, 1755, 1604, 1492, 1454, 1402, 1238, 1092, 1029, 917 $\mathrm{cm}^{-1} ;{ }^{1} \mathrm{H}$ NMR $\left(500 \mathrm{MHz}, \mathrm{CDCl}_{3}\right) \delta 7.38(\mathrm{~d}, J=8.0,2 \mathrm{H}), 7.34(\mathrm{dd}, J=7.8,7.8 \mathrm{~Hz}, 2 \mathrm{H}), 7.28(\mathrm{~d}, J=7.6$ $\mathrm{Hz}, 1 \mathrm{H}), 7.24(\mathrm{dd}, J=7.6,7.6 \mathrm{~Hz}, 2 \mathrm{H}), 7.19(\mathrm{dd}, J=7.0,7.0 \mathrm{~Hz}, 1 \mathrm{H}), 6.97(\mathrm{~d}, J=7.6,2 \mathrm{H}), 4.52(\mathrm{~s}$, $1 \mathrm{H}), 3.90(\mathrm{~d}, J=7.8 \mathrm{~Hz}, 1 \mathrm{H}), 3.79(\mathrm{dd}, J=8.8,3.9 \mathrm{~Hz}, 1 \mathrm{H}), 3.73(\mathrm{dd}, J=8.5,8.5 \mathrm{~Hz}, 1 \mathrm{H}), 3.22(\mathrm{dd}, J=$ 13.4, 3.4 Hz, 1H), 3.05 (dddd, $J=10.7,7.4,3.5,3.5 \mathrm{~Hz}, 1 \mathrm{H}), 2.46$ (dd, $J=13.4,10.0 \mathrm{~Hz}, 1 \mathrm{H}), 2.04-1.94$ $(\mathrm{m}, 1 \mathrm{H}), 1.10(\mathrm{~d}, J=6.6 \mathrm{~Hz}, 3 \mathrm{H}), 0.75(\mathrm{~d}, J=6.8 \mathrm{~Hz}, 3 \mathrm{H}) ;{ }^{13} \mathrm{C} \mathrm{NMR}\left(125 \mathrm{MHz}, \mathrm{CDCl}_{3}\right) \delta 157.7$, 141.3, 136.0, 129.1, 128.7, 128.1, 127.6, 126.9, 70.6, 65.6, 58.1, 36.6, 32.4, 19.8, 19.1; MS (CI) m/z (relative intensity) $325\left([\mathrm{M}+\mathrm{H}]^{+}, 6 \%\right), 193(100 \%), 133(27 \%)$; Anal. Calcd for $\mathrm{C}_{20} \mathrm{H}_{24} \mathrm{~N}_{2} \mathrm{O}_{2}$ : C, 74.04; H, 7.46; N, 8.63. Found: C, 74.24; H, 7.62; N, 8.48.

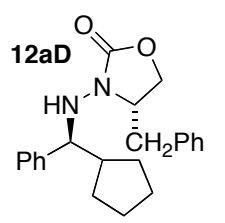

(4S,1'S)-3-(1'-Cyclopentyl-1'-phenylmethylamino)-4-phenylmethyl-2-

oxazolidinone $[(S, S)-12 a D]$. From $7(42 \mathrm{mg}, 0.150 \mathrm{mmol})$ and iodocyclopentane by General Procedure $\mathrm{C}$ was obtained $(\boldsymbol{S}, \boldsymbol{S}) \mathbf{- 1 2 a D}(31 \mathrm{mg}, 59 \%$ yield, S,S/S,R $=96: 4)$ as a colorless oil. GCMS retention time $5.5 \mathrm{~min} ;[\alpha]_{\mathrm{D}}{ }^{26}-11.7^{\circ},\left(c 1.1, \mathrm{CHCl}_{3}\right)$; IR (film) 3302 , 3061, 3027, 2953, 2868, 1756, 1603, 1489, 1403, 1289, 1007, $922 \mathrm{~cm}^{-1} ;{ }^{1} \mathrm{H}$ NMR (500 $\left.\mathrm{MHz}, \mathrm{CDCl}_{3}\right) \delta 7.42(\mathrm{dd}, J=8.5,1.4 \mathrm{~Hz}, 2 \mathrm{H}), 7.36(\mathrm{dd}, J=7.1,7.1 \mathrm{~Hz}, 2 \mathrm{H}), 7.29$ (dddd, $J=7.4,7.4$, $1.4,1.4 \mathrm{~Hz}, 1 \mathrm{H}), 7.25$ (dddd, $J=7.0,7.0,1.5,1.5 \mathrm{~Hz}, 2 \mathrm{H}), 7.20$ (dddd, $J=7.3,7.3,1.1,1.1 \mathrm{~Hz}, 1 \mathrm{H})$, $6.96(\mathrm{~d}, J=7.1 \mathrm{~Hz}, 2 \mathrm{H}), 4.54(\mathrm{~s}, 1 \mathrm{H}), 3.93(\mathrm{~d}, J=9.8 \mathrm{~Hz}, 1 \mathrm{H}), 3.80(\mathrm{dd}, J=8.8,4.1 \mathrm{~Hz}, 1 \mathrm{H}), 3.75(\mathrm{dd}, J$ $=7.8,7.8 \mathrm{~Hz}, 1 \mathrm{H}), 3.21(\mathrm{dd}, J=13.4,3.3 \mathrm{~Hz}, 1 \mathrm{H}), 2.99$ (dddd, $J=10.0,7.3,3.7,3.7 \mathrm{~Hz}, 1 \mathrm{H}), 2.46(\mathrm{dd}, J$ $=13.4,10.0 \mathrm{~Hz}, 1 \mathrm{H}), 2.17(\mathrm{~m}$, apparent sextet, $J=8.5 \mathrm{~Hz}, 1 \mathrm{H})$, , 2.07-1.99 (m, 1H), 1.80-1.73 $(\mathrm{m}, 1 \mathrm{H})$, 1.69-1.54 (m, 3H), 1.49-1.41 (m, 1H), 1.36-1.29 (m, 1H), 1.15-1.07 (m, 1H); ${ }^{13} \mathrm{C} \mathrm{NMR}(125 \mathrm{MHz}$, $\left.\mathrm{CDCl}_{3}\right) \delta 157.6,142.6,136.0,129.1,128.7,128.3,128.2,127.6,126.8,70.2,65.6,58.1,44.6,36.5,30.2$, 30.1, 25.7, 24.9; MS (CI) $\mathrm{m} / z$ (relative intensity) $351\left([\mathrm{M}+\mathrm{H}]^{+}, 11 \%\right), 193(100 \%)$; Anal. Calcd for $\mathrm{C}_{22} \mathrm{H}_{26} \mathrm{~N}_{2} \mathrm{O}_{2}$ : C, 75.40; H, 7.48; N, 7.99. Found: C, 75.29; H, 7.53; N, 7.83.

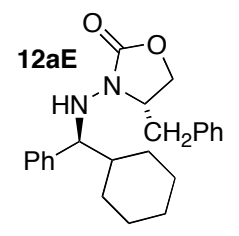

(4S,1'S)-3-(1'-Cyclohexyl-1'-phenylmethylamino)-4-phenylmethyl-2-

oxazolidinone $[(S, S)-12 a E]$. From $7(79 \mathrm{mg}, 0.282 \mathrm{mmol})$ and iodocyclohexane by General Procedure $\mathrm{C}$ was obtained $(\boldsymbol{S}, \boldsymbol{S}) \mathbf{- 1 2 a E}(32 \mathrm{mg}, 30 \%$ yield, $\mathrm{S}, \mathrm{S} / \mathrm{S}, \mathrm{R}=99: 1)$ as a colorless oil. GCMS retention time $9.7 \mathrm{~min} ;[\alpha]_{\mathrm{D}}{ }^{26}-16.2^{\circ}\left(c 0.55, \mathrm{CHCl}_{3}\right)$; IR (film) $3309,3027,2925,2852,1755,1695,1603,1490,1403,1219,1028,893 \mathrm{~cm}^{-1} ;{ }^{1} \mathrm{H}$ NMR $\left(500 \mathrm{MHz}, \mathrm{CDCl}_{3}\right) \delta 7.39(\mathrm{~d}, J=7.0,2 \mathrm{H}), 7.35(\mathrm{dd}, J=7.3,7.3 \mathrm{~Hz}, 2 \mathrm{H}), 7.30(\mathrm{~d}, J=6.8 \mathrm{~Hz}, 1 \mathrm{H}), 7.26$ $(\mathrm{dd}, J=7.0,7.0 \mathrm{~Hz}, 2 \mathrm{H}), 7.20(\mathrm{dd}, J=7.1,7.1 \mathrm{~Hz}, 1 \mathrm{H}), 6.98(\mathrm{~d}, J=7.1 \mathrm{~Hz}, 2 \mathrm{H}), 4.59(\mathrm{~s}, 1 \mathrm{H}), 3.95(\mathrm{~d}, J$ $=7.0 \mathrm{~Hz}, 1 \mathrm{H}), 3.81(\mathrm{dd}, J=8.9,4.0 \mathrm{~Hz}, 1 \mathrm{H}), 3.74(\mathrm{dd}, J=7.9,7.9 \mathrm{~Hz}, 1 \mathrm{H}), 3.22(\mathrm{dd}, J=13.4,3.1 \mathrm{~Hz}$, $1 \mathrm{H}), 3.07-3.02(\mathrm{~m}, 1 \mathrm{H}), 2.47(\mathrm{dd}, J=13.2,10.0 \mathrm{~Hz}, 1 \mathrm{H}), 2.03(\mathrm{~d}, J=12.4 \mathrm{~Hz}, 1 \mathrm{H}), 1.81(\mathrm{~d}, J=13.2 \mathrm{~Hz}$, 
$1 \mathrm{H}), 1.71-1.63(\mathrm{~m}, 3 \mathrm{H}), 1.40(\mathrm{~d}, J=12.9 \mathrm{~Hz}, 1 \mathrm{H}), 1.34-1.26(\mathrm{~m}, 1 \mathrm{H}), 1.20-1.11(\mathrm{~m}, 3 \mathrm{H}), 0.92-0.84(\mathrm{~m}$, $1 \mathrm{H}) ;{ }^{13} \mathrm{C}$ NMR $\left(125 \mathrm{MHz}, \mathrm{CDCl}_{3}\right) \delta$ 157.6, 141.5, 136.1, 129.1, 128.7, 128.1, 127.6, 126.9, 69.8, 65.5, 58.2, 41.9, 36.6, 30.2, 29.3, 26.3, 26.0; MS (CI) $\mathrm{m} / \mathrm{z}$ (relative intensity) $365\left([\mathrm{M}+\mathrm{H}]^{+}, 6 \%\right), 193(100 \%)$; 173 (34\%); Anal. Calcd for $\mathrm{C}_{23} \mathrm{H}_{28} \mathrm{~N}_{2} \mathrm{O}_{2}$ : C, 75.79; H, 7.74; N, 7.69. Found: C, 76.04; H, 7.92; N, 7.43.

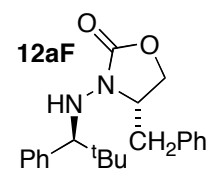

(4S,1'S)-3-(2',2'-Dimethyl-1'-phenyl-1'-propylamino)-4-phenylmethyl-2oxazolidinone $[(\boldsymbol{S}, \boldsymbol{S}) \mathbf{- 1 2} \mathbf{a F}]$. From 7 (51 $\mathrm{mg}, 0.182 \mathrm{mmol})$ and 2-iodo-2,2dimethylpropane by General Procedure $C$ was obtained $(S, S)-\mathbf{1 2 a F}(51 \mathrm{mg}, 83 \%$ yield, $\mathrm{S}, \mathrm{S} / \mathrm{S}, \mathrm{R}=93: 7)$ as a colorless solid. GCMS retention time $6.1 \mathrm{~min} ; \mathrm{mp} 84-85^{\circ} \mathrm{C} ;[\alpha]_{\mathrm{D}}{ }^{26}$ $-35.8^{\circ}$ (c 1.6, $\mathrm{CHCl}_{3}$ ); IR (film) 3317, 3062, 2955, 1755, 1604, 1492, 1454, 1366, 1222, 1094, $963 \mathrm{~cm}^{-1}$; ${ }^{1} \mathrm{H}$ NMR $\left(500 \mathrm{MHz}, \mathrm{CDCl}_{3}\right) \delta 7.42$ (very br s, 2H), 7.34-7.23 (m, 5H), $7.20(\mathrm{dd}, J=7.4,7.4 \mathrm{~Hz}, 1 \mathrm{H})$, $7.03(\mathrm{~d}, J=7.3 \mathrm{~Hz}, 2 \mathrm{H}), 4.58(\mathrm{~s}, 1 \mathrm{H}), 3.97(\mathrm{~s}, 1 \mathrm{H}), 3.81(\mathrm{dd}, J=8.8,3.4 \mathrm{~Hz}, 1 \mathrm{H}), 3.74(\mathrm{dd}, J=8.1,8.1$ $\mathrm{Hz}, 1 \mathrm{H}), 3.29$ (dd, $J=13.4,3.2 \mathrm{~Hz}, 1 \mathrm{H}), 3.22$ (dddd, $J=9.7,7.6,4.1,4.1 \mathrm{~Hz}, 1 \mathrm{H}), 2.51(\mathrm{dd}, J=13.3$, $9.9 \mathrm{~Hz}, 1 \mathrm{H}), 1.0(\mathrm{~s}, 9 \mathrm{H}) ;{ }^{13} \mathrm{C} \mathrm{NMR}\left(125 \mathrm{MHz}, \mathrm{CDCl}_{3}\right) \delta 157.7,140.3,136.1,129.1,128.7,127.5,126.9$, 73.5, 65.4, 58.1, 36.7, 34.1, 26.9; MS (CI) $\mathrm{m} / z$ (relative intensity) $339\left([\mathrm{M}+\mathrm{H}]^{+}, 16 \%\right), 193(100 \%), 147$ (17\%); Anal. Calcd for $\mathrm{C}_{21} \mathrm{H}_{26} \mathrm{~N}_{2} \mathrm{O}_{2}:$ C, 74.52; H, 7.74; N, 8.28. Found: C, 74.40; H, 7.79; N, 8.24.

\section{Stereochemical Proof of 9aC by Chemical Correlation}
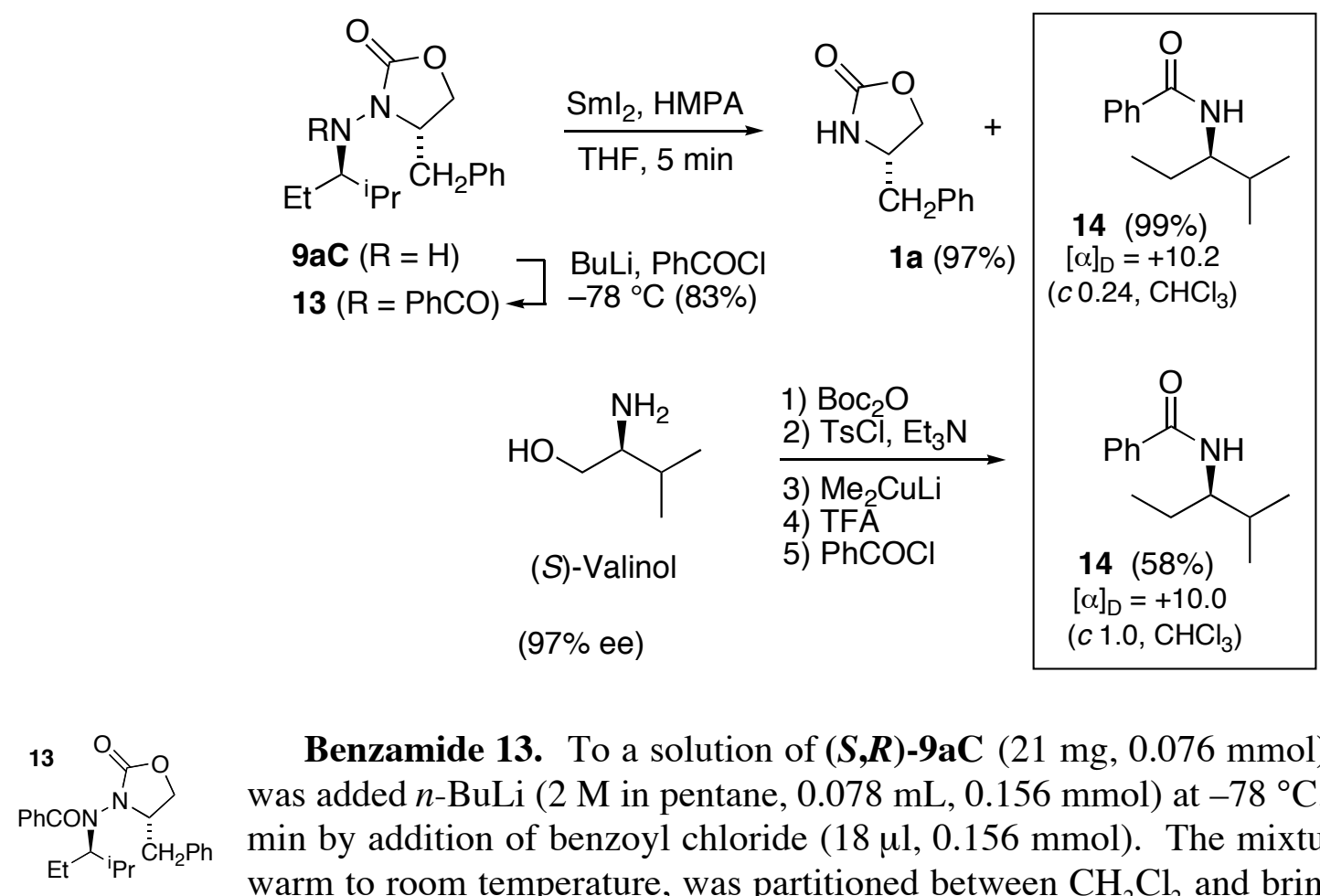

Benzamide 13. To a solution of $(\boldsymbol{S}, \boldsymbol{R})-9 \mathrm{aC}(21 \mathrm{mg}, 0.076 \mathrm{mmol})$ in THF $(0.5 \mathrm{~mL})$ was added $n$-BuLi ( $2 \mathrm{M}$ in pentane, $0.078 \mathrm{~mL}, 0.156 \mathrm{mmol}$ ) at $-78{ }^{\circ} \mathrm{C}$, followed after 30 min by addition of benzoyl chloride $(18 \mu 1,0.156 \mathrm{mmol})$. The mixture was allowed to warm to room temperature, was partitioned between $\mathrm{CH}_{2} \mathrm{Cl}_{2}$ and brine, and the organic phase was dried over $\mathrm{Na}_{2} \mathrm{SO}_{4}$. Concentration and gradient flash chromatography (hexane $\rightarrow$ 3:1 hexane/EtOAc) afforded 13 (24 mg, 83\% yield), which exhibited line broadening in ${ }^{1} \mathrm{H}$ NMR spectra due to restricted amide bond rotation. Colorless solid; mp $139-140{ }^{\circ} \mathrm{C} ;[\alpha]_{\mathrm{D}}{ }^{27}+20.8^{\circ}\left(c 0.61, \mathrm{CHCl}_{3}\right)$; IR (film) 3062, 3028, 2965, 2876, 1783, 1660, 1495, 1395, 1337, 1194, $913 \mathrm{~cm}^{-1}$; ${ }^{1} \mathrm{H}$ NMR (500 MHz, $\left.\mathrm{C}_{7} \mathrm{D}_{8}, 363 \mathrm{~K}\right) \delta 7.48$ (br s, 2H), 7.06-6.96 (m, 6H), $6.80(\mathrm{~s}, 2 \mathrm{H}), 4.20-3.49$ (very br s, 2H), $3.61(\mathrm{~s}, 1 \mathrm{H})$ 3.53-3.49 (m, 1H), 3.11 (d, $J=13.6 \mathrm{~Hz}, 1 \mathrm{H}), 2.46$ (br s, 1H), 2.09 (br s, 1H), 1.83 (br s, 1H), 1.62 (br s, 1H), 1.00 (br s, 6H), 0.89 (d, $J=5.3 \mathrm{~Hz}, 3 \mathrm{H}) ;{ }^{13} \mathrm{C} \mathrm{NMR}\left(125 \mathrm{MHz}, \mathrm{CDCl}_{3}\right) \delta 171.5,156.1,136.2,135.8$, 
$135.4,129.8,129.0,128.9,128.5,128.3,127.3,126.6,70.4,68.1,67.6,65.8,61.0,58.9,39.7,36.8,31.4$, 22.9, 21.9, 21.7, 19.9, 18.2, 12.2, 12.0; MS (CI) $\mathrm{m} / z$ (relative intensity) $381\left([\mathrm{M}+\mathrm{H}]^{+}, 56 \%\right), 297(65 \%)$, $105(100 \%)$; Anal. Calcd for $\mathrm{C}_{23} \mathrm{H}_{28} \mathrm{~N}_{2} \mathrm{O}_{3}$ : C, 72.06; H, 7.42; N, 7.36. Found: C, 72.60; H, 7.49; N, 7.36. ${ }^{2}$

14 NHCOPh $(\boldsymbol{R})-2-M e t h y l p e n t-3-y l$ benzamide (14). To a solution of 13 (20.9 $\mathrm{mg}, 0.055 \mathrm{mmol})$ ${ }_{\text {Et }}{ }_{\text {iPr }}$ in THF $(0.7 \mathrm{~mL})$ and HMPA $(30 \mu \mathrm{l})$ at $-78{ }^{\circ} \mathrm{C}$ was added $\mathrm{SmI}_{2}(0.25 \mathrm{M}$ in THF $)$ until the solution turned deep blue. After $5 \mathrm{~min}$, TLC indicated complete conversion. The mixture was partitioned between $\mathrm{CH}_{2} \mathrm{Cl}_{2}$ and brine, and the organic phase was dried over $\mathrm{Na}_{2} \mathrm{SO}_{4}$. Concentration and flash chromatography afforded $\mathbf{1 a}(9.5 \mathrm{mg}, 97 \%$ yield) and $\mathbf{1 4}(11.2 \mathrm{mg}, 99 \%$ yield $)$ as a colorless solid; mp 116-117 ${ }^{\circ} \mathrm{C}$; $[\alpha]_{\mathrm{D}}{ }^{26}+10.2^{\circ}\left(c 0.24, \mathrm{CHCl}_{3}\right)$; IR (film) 3292, 3059, 2960, 2938, 2873, 1633, 1578, 1542, 1491, 1460, 1368, 1158, $985 \mathrm{~cm}^{-1} ;{ }^{1} \mathrm{H}$ NMR $\left(500 \mathrm{MHz}, \mathrm{CDCl}_{3}\right) \delta 7.74$ (ddd, $J=6.9,1.5,1.5$ $\mathrm{Hz}, 2 \mathrm{H}$ ), 7.47 (dddd, $J=7.3,7.3,1.4,1.4 \mathrm{~Hz}, 1 \mathrm{H}), 7.41$ (dddd, $J=7.1,7.1,1.4,1.4 \mathrm{~Hz}, 2 \mathrm{H}$ ), 5.77 (br s, $1 \mathrm{H}), 3.97-3.91(\mathrm{~m}, 1 \mathrm{H}), 1.89-1.80(\mathrm{~m}, 1 \mathrm{H}), 1.70-1.62(\mathrm{~m}, 1 \mathrm{H}), 1.45-1.36(\mathrm{~m}, 1 \mathrm{H}), 0.96-0.93(\mathrm{~m}, 9 \mathrm{H})$; ${ }^{13} \mathrm{C}$ NMR $\left(125 \mathrm{MHz}, \mathrm{CDCl}_{3}\right) \delta 167.5,135.3,131.2,128.6,126.8,56.1,31.8,25.3,19.4,17.9,10.6$; MS (CI) $m / z$ (relative intensity) $206\left([\mathrm{M}+\mathrm{H}]^{+}, 100 \%\right.$ ); Anal. Calcd for $\mathrm{C}_{13} \mathrm{H}_{19} \mathrm{NO}$ : C, 76.05; H, 9.33; N, 6.82. Found: C, 75.98; H, 9.24; N, 6.71.

\section{Stereochemical Proof of 12aF by Crystallography}<smiles>O=C1OCC(Br)N1NC(Br)c1ccccc1</smiles>

$12 \mathrm{aF}$

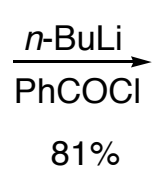

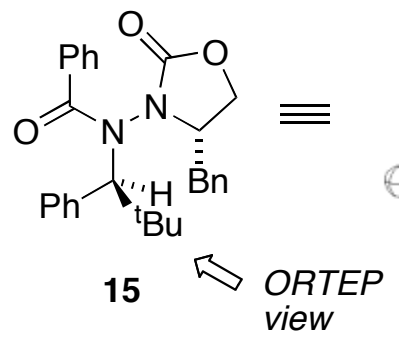
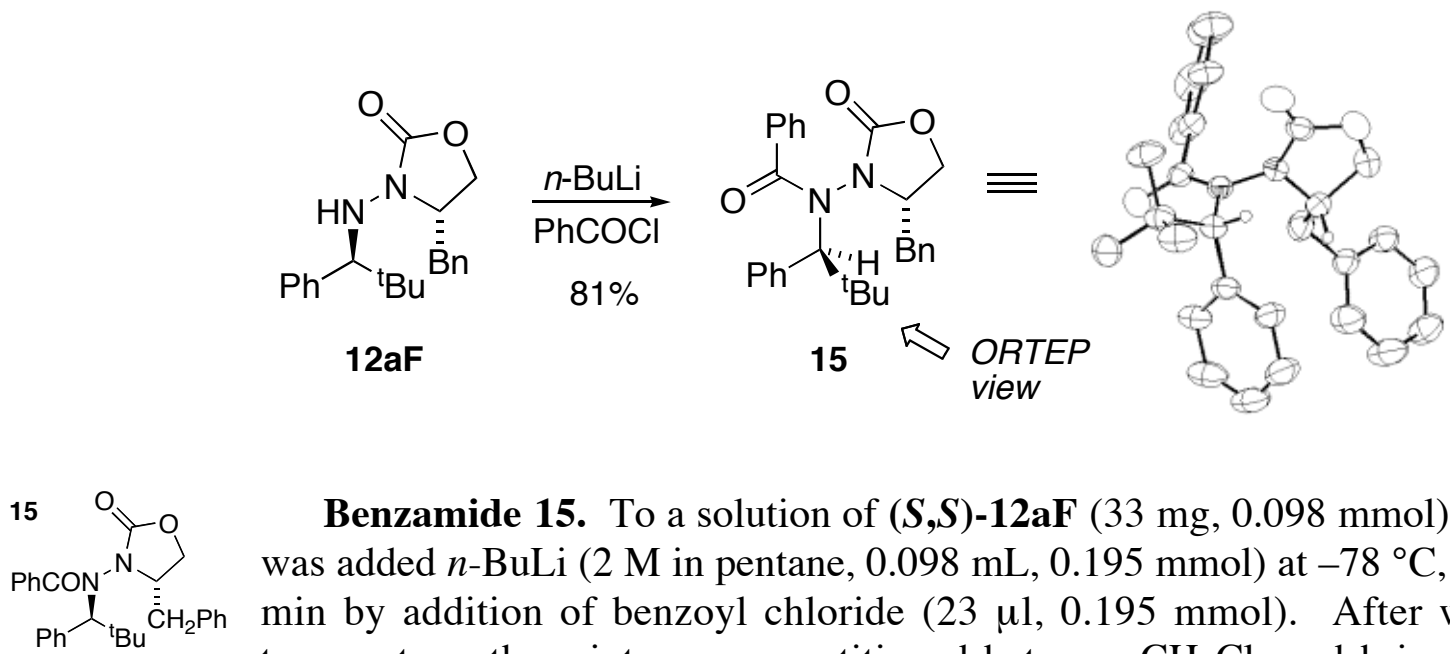

Benzamide 15. To a solution of $(\boldsymbol{S}, \boldsymbol{S})$-12aF (33 $\mathrm{mg}, 0.098 \mathrm{mmol})$ in THF (1.0 mL) was added $n$-BuLi ( $2 \mathrm{M}$ in pentane, $0.098 \mathrm{~mL}, 0.195 \mathrm{mmol}$ ) at $-78{ }^{\circ} \mathrm{C}$, followed after 40 min by addition of benzoyl chloride $(23 \mu \mathrm{l}, 0.195 \mathrm{mmol})$. After warming to room temperature, the mixture was partitioned between $\mathrm{CH}_{2} \mathrm{Cl}_{2}$ and brine, and the organic phase was dried over $\mathrm{Na}_{2} \mathrm{SO}_{4}$. Concentration and gradient flash chromatography (hexane $\rightarrow 3: 1$ hexane/EtOAc) afforded 15 (35 mg, 81\% yield), which exhibited line broadening in ${ }^{1} \mathrm{H}$ NMR spectra due to restricted amide bond rotation. Recrystallization from 95\% EtOH and 3:1 hexane/EtOAc afforded colorless orthorhombic crystals; mp $199-199.5^{\circ} \mathrm{C} ;[\alpha]_{\mathrm{D}}{ }^{27}+47.7^{\circ}\left(c 0.65, \mathrm{CHCl}_{3}\right)$; IR (film) 3064, 3028, 2962, 1775, 1668, 1447, 1397, 1199, 1109, 1029, $912 \mathrm{~cm}^{-1} ;{ }^{1} \mathrm{H}$ NMR (500 MHz, CDCl $\left.{ }_{3}\right)$ $\delta 7.79(\mathrm{~s}, 1 \mathrm{H}), 7.69(\mathrm{~d}, J=7.3 \mathrm{~Hz}, 2 \mathrm{H}), 7.47-7.37(\mathrm{~m}, 7 \mathrm{H}), 7.08-7.03(\mathrm{~m}, 3 \mathrm{H}), 6.17(\mathrm{~s}, 2 \mathrm{H}), 4.16(\mathrm{~s}, 1 \mathrm{H})$, 3.80-3.62 (m, 2H), 3.50-3.40 (m, 1H), 2.69-2.60 (m, 1H), 1.65-1.50 (m, 1H), $1.27(\mathrm{~s}, 9 \mathrm{H}) ;{ }^{13} \mathrm{C} \mathrm{NMR}$ $\left(125 \mathrm{MHz}, \mathrm{CDCl}_{3}\right) \mathrm{d} 174.6,156.1,141.3,136.5,134.2,130.5,130.2,128.7,128.3,127.4,127.1,81.4$, 67.6, 59.2, 37.1, 36.8, 29.1; MS (CI) $\mathrm{m} / z$ (relative intensity) $443\left([\mathrm{M}+\mathrm{H}]^{+}, 33 \%\right), 297(100 \%)$; Anal. Calcd for $\mathrm{C}_{28} \mathrm{H}_{30} \mathrm{~N}_{2} \mathrm{O}_{3}:$ C, 75.99; H, 6.83; N, 6.33. Found: C, 75.32; H, 6.89; N, 6.24. ${ }^{2}$ The assigned structure was further confirmed by $\mathrm{x}$-ray crystallography. 


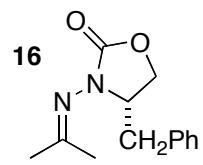

(S)-3-(2-Propylidene)amino-4-phenylmethyl-2-oxazolidinone (16). From 3a (60 $\mathrm{mg}, 0.259 \mathrm{mmol}$ ) and 2-iodopropane, by General Procedure E, photolytic radical addition in the presence of acetone gave the isopropyl adduct $\mathbf{9 a C}$ (36 mg, $50 \%$ yield) and $\mathbf{1 6}$ (20 $\mathrm{mg}, 33 \%$ yield) as a colorless oil; $[\alpha]_{\mathrm{D}}{ }^{25}-65.9^{\circ}\left(c 0.94, \mathrm{CHCl}_{3}\right)$; IR (film) 3061, 3028, 2999, 2921, 1764, 1643, 1395, 1365, 1219, 1094, $1044 \mathrm{~cm}^{-1} ;{ }^{1} \mathrm{H}$ NMR $\left(500 \mathrm{MHz}, \mathrm{CDCl}_{3}\right) \delta$ 7.31-7.13 $(\mathrm{m}, 5 \mathrm{H}), 4.36-4.29(\mathrm{~m}, 1 \mathrm{H}), 4.25(\mathrm{dd}$, apparent $\mathrm{t}, J=8.5 \mathrm{~Hz}, 1 \mathrm{H}), 4.04(\mathrm{dd}$, apparent $\mathrm{t}, J=9.0 \mathrm{~Hz}, 1 \mathrm{H})$, $3.15(\mathrm{dd}, J=13.7,4.1 \mathrm{~Hz}, 1 \mathrm{H}), 2.73(\mathrm{dd}, J=13.6,8.9 \mathrm{~Hz}, 1 \mathrm{H}), 2.12(\mathrm{~s}, 3 \mathrm{H}), 2.02(\mathrm{~s}, 3 \mathrm{H}) ;{ }^{13} \mathrm{C} \mathrm{NMR}$ $\left(125 \mathrm{MHz}, \mathrm{CDCl}_{3}\right) \delta 173.7,154.6,135.5,129.1,128.7,127.0,66.6,60.6,38.4,25.2,20.7$; MS (CI) $\mathrm{m} / z$ (relative intensity) $233\left([\mathrm{M}+\mathrm{H}]^{+}, 43 \%\right), 193(100 \%)$. The ${ }^{1} \mathrm{H}$ NMR spectrum was identical to an authentic sample prepared from $\mathbf{2 a}$ and acetone.

\section{References and Notes}

(1) CAUTION: $O$-(Mesitylenesulfonyl)hydroxylamine $\left(\mathrm{MtsONH}_{2}\right)$ shows a tendency toward exothermic spontaneous decomposition during its preparation and storage. Although most of the work described here was conducted using $\mathrm{MtsONH}_{2}$, we have discontinued use of this compound in favor of the alternative amination reagent $O$-( $p$-nitrobenzoyl $)$ hydroxylamine $\left(\mathrm{NbzONH}_{2}\right)$, which gives similar results in the amination of oxazolidinones. Another viable alternative is $O$ (diphenylphosphinoyl)hydroxylamine $\left(\mathrm{DppONH}_{2}\right)$.

(2) Slight deviation from theory was noted in combustion analyses of $\mathbf{2 a}, \mathbf{1 3}$, and $\mathbf{1 5}$. Unfortunately, samples for further analysis were not available at the time of manuscript preparation.

(3) Shen, Y.; Friestad, G. K. J. Org. Chem. 2002, 67, 6236-6239.

(4) Cook, G. R.; Maity, B. C.; Kargbo, R. Org. Lett. 2004, 6, 1741-1743.

(5) Friestad, G. K.; Ding, H. Angew. Chem. Int. Ed. 2001, 40, 4491-4493.

(6) Qin, J.; Friestad, G. K. Tetrahedron 2003, 59, 6393-6402. 


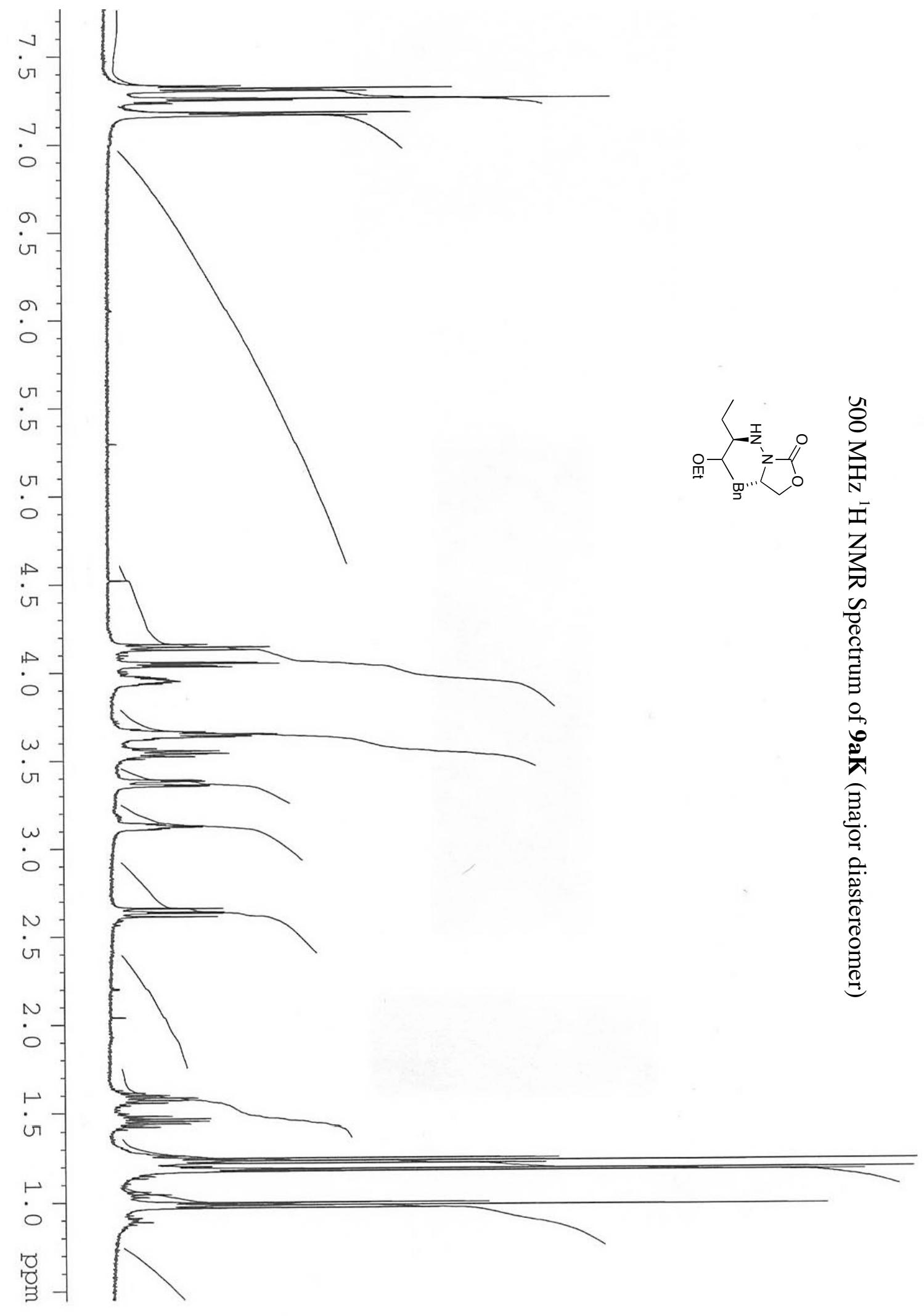




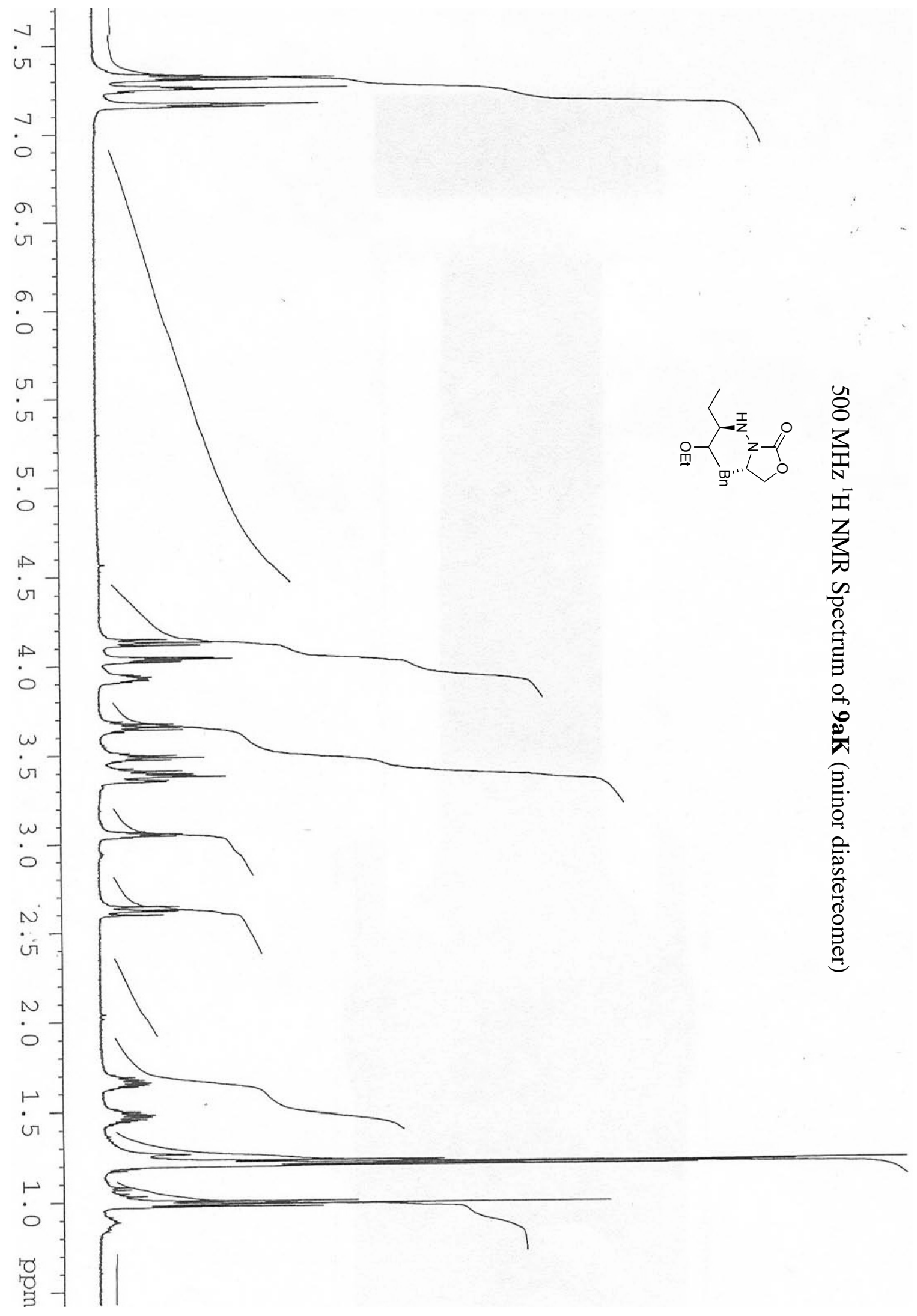




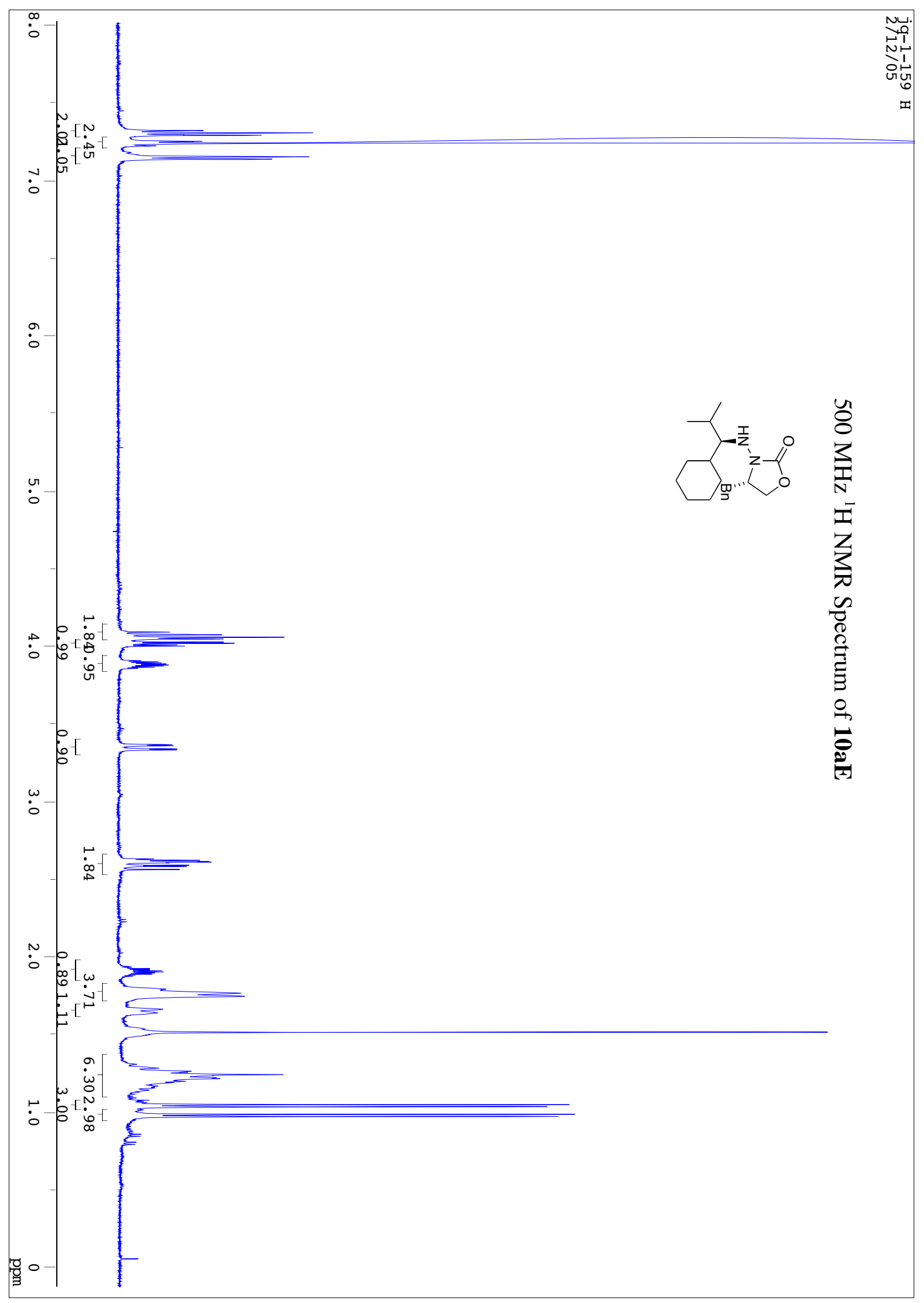




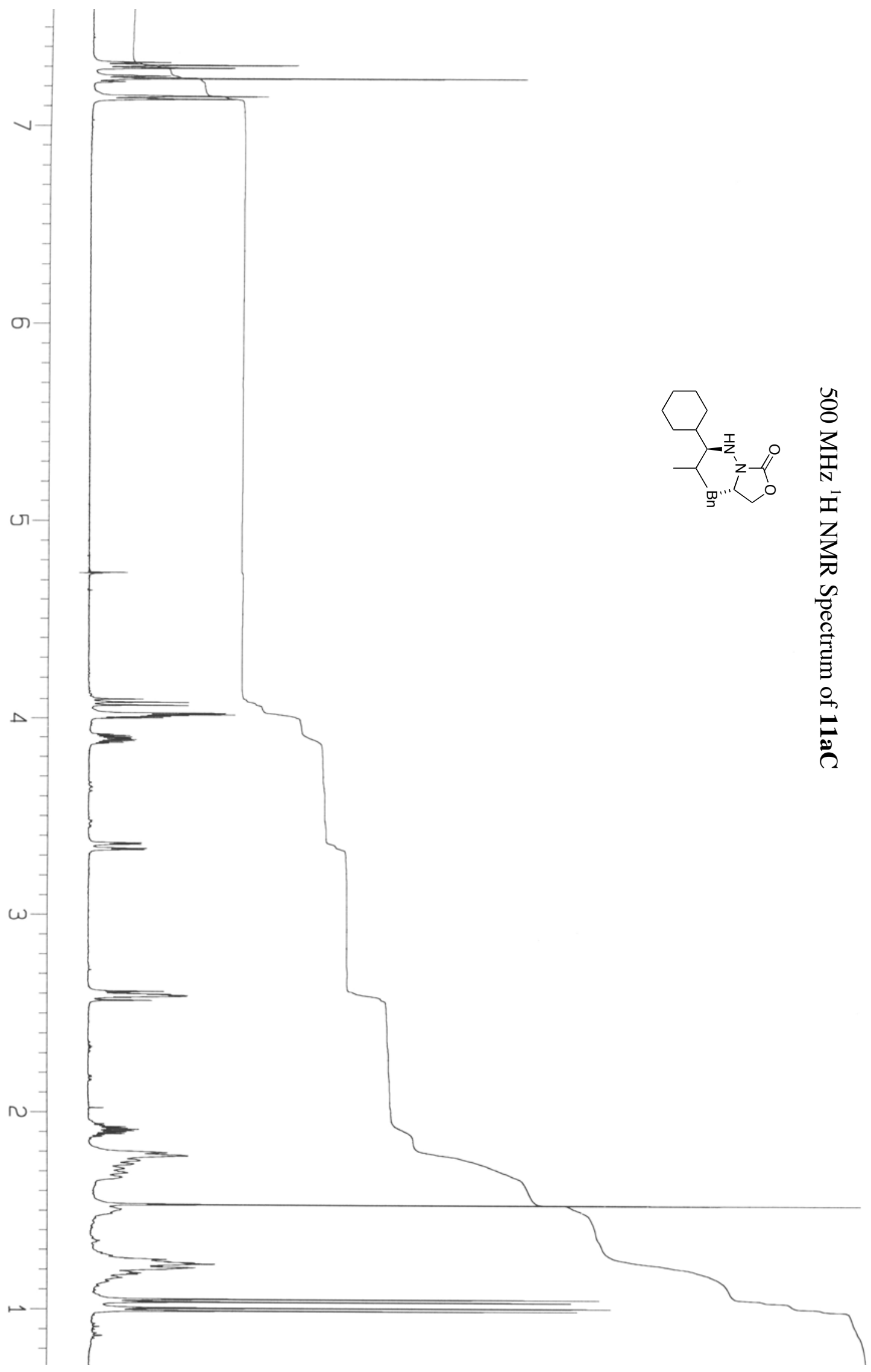




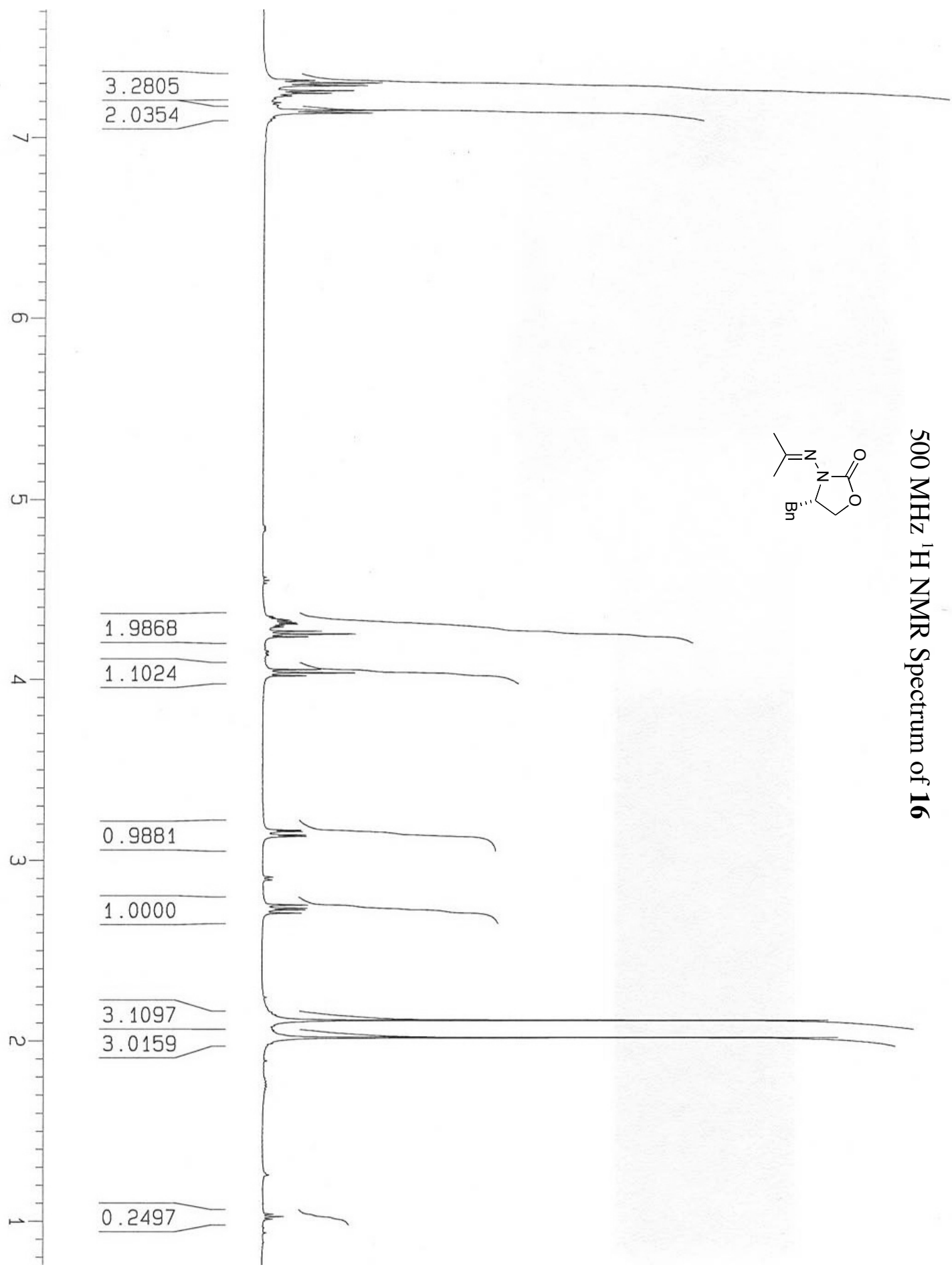




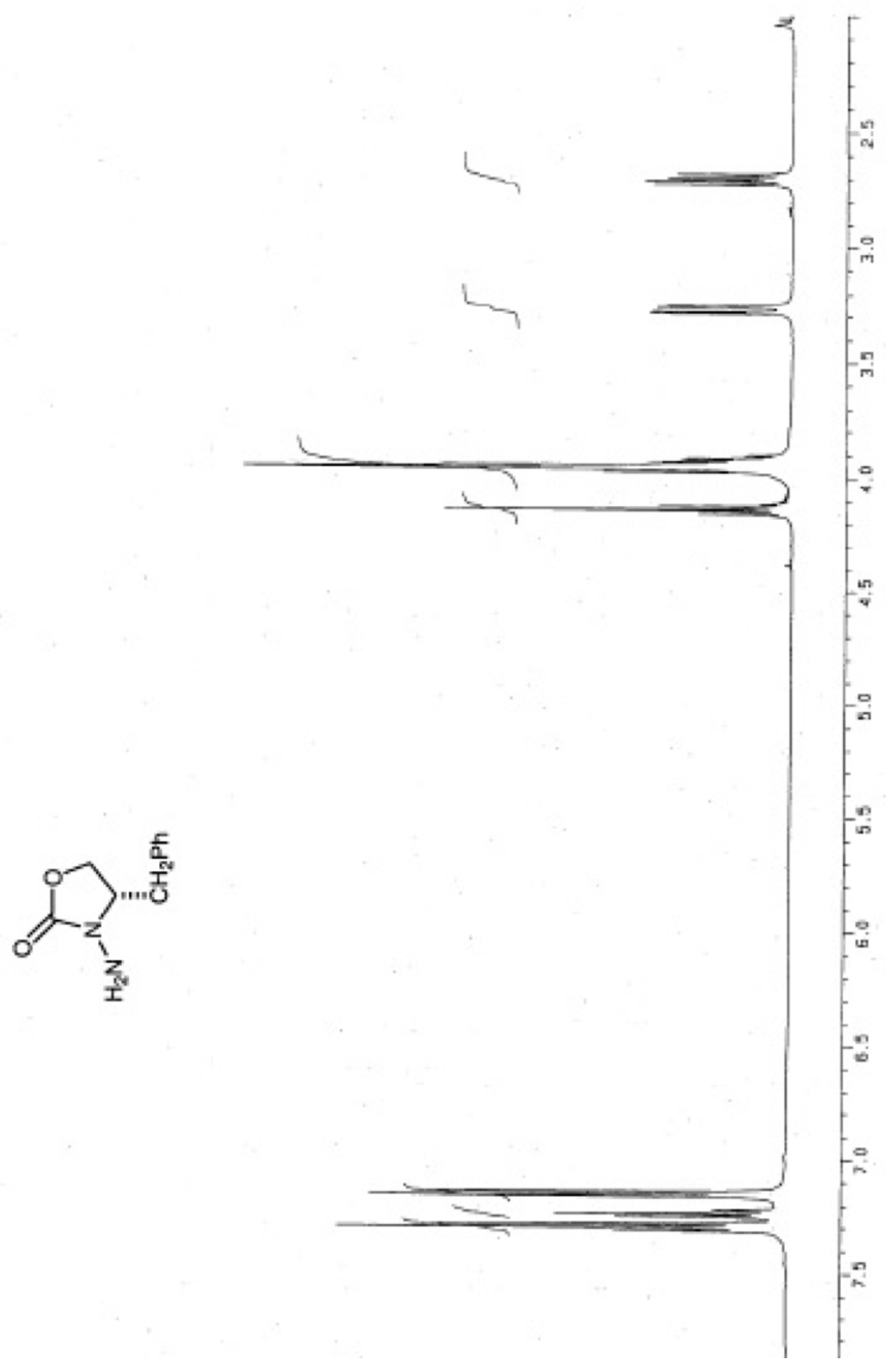




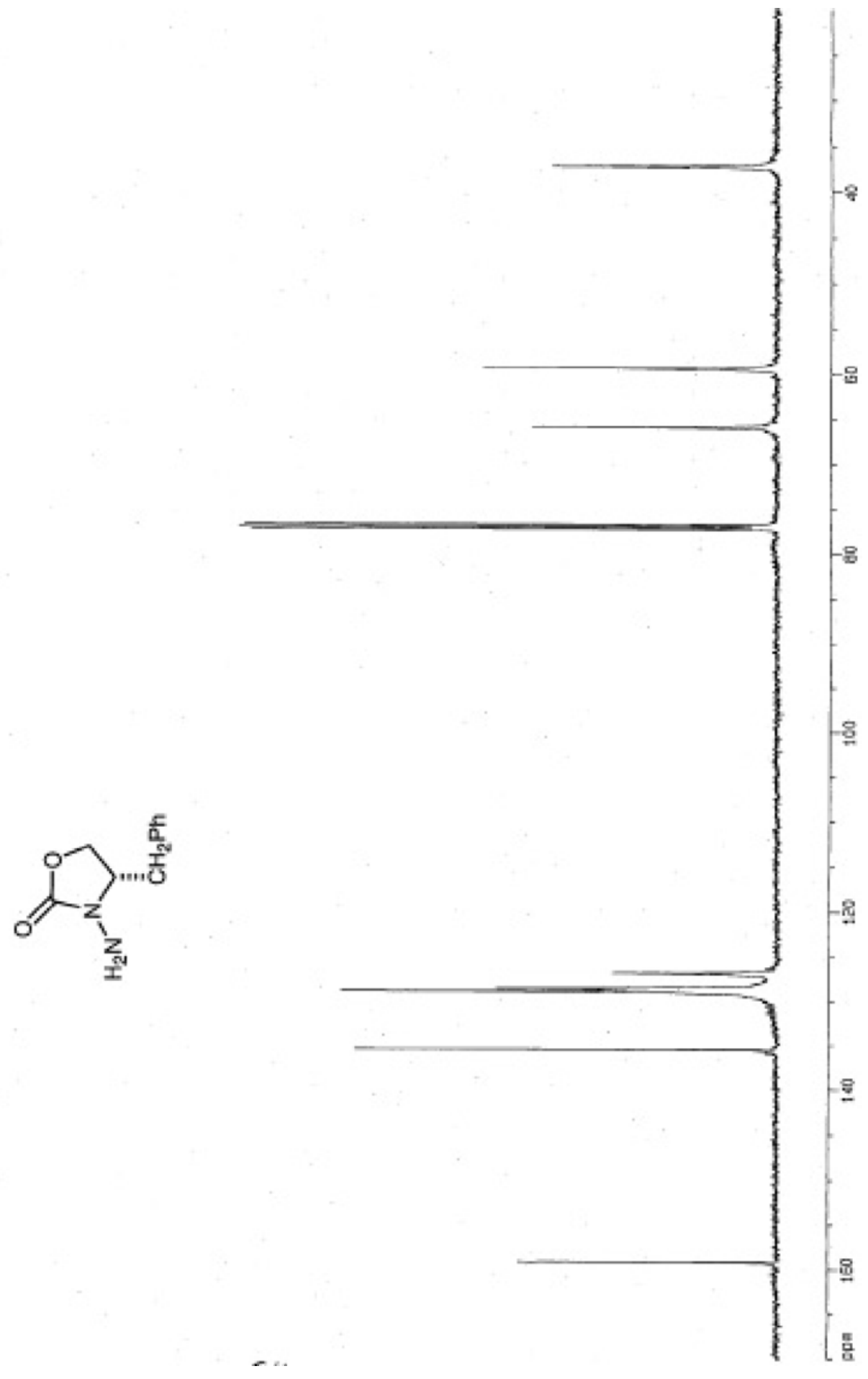




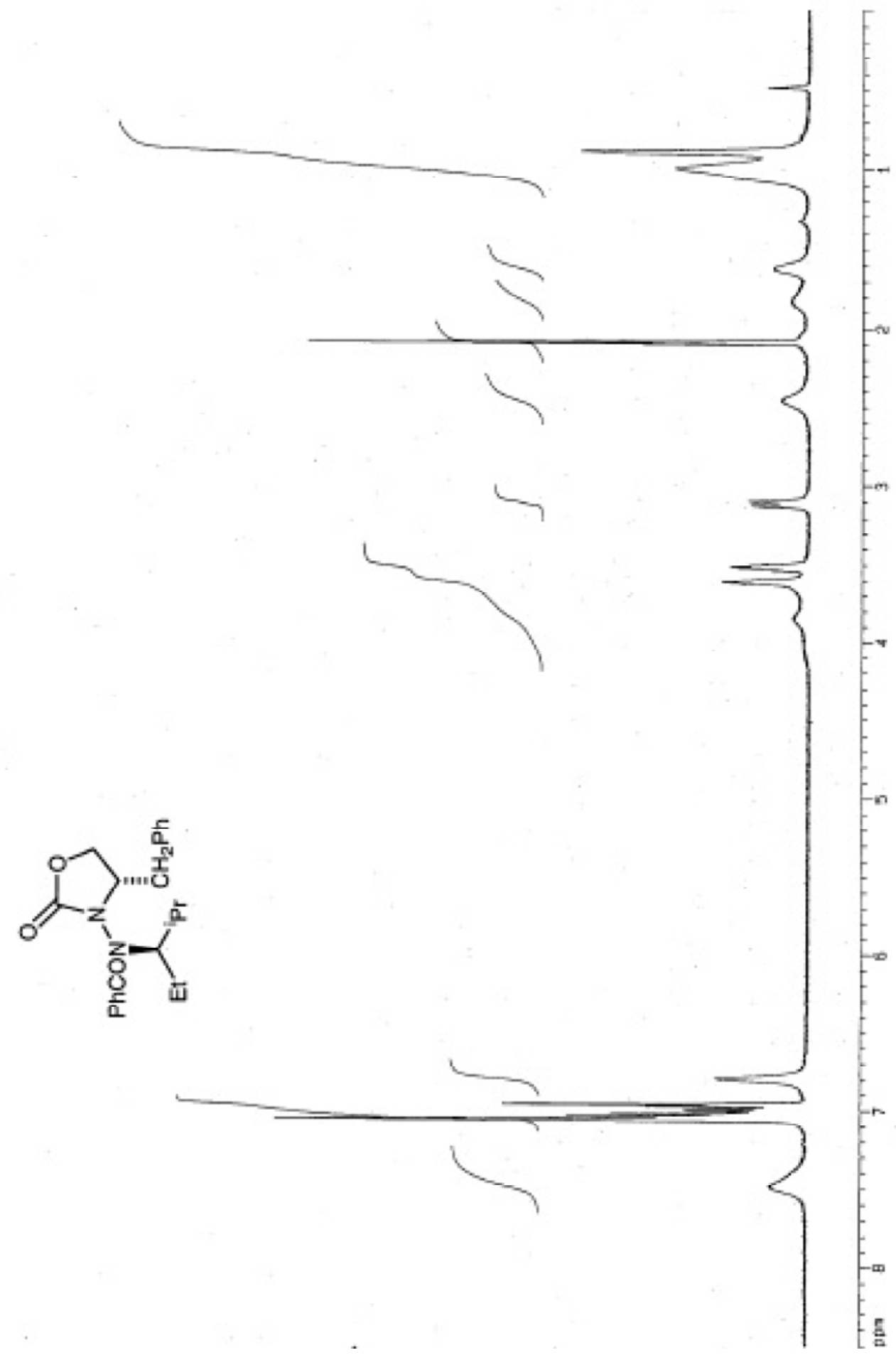




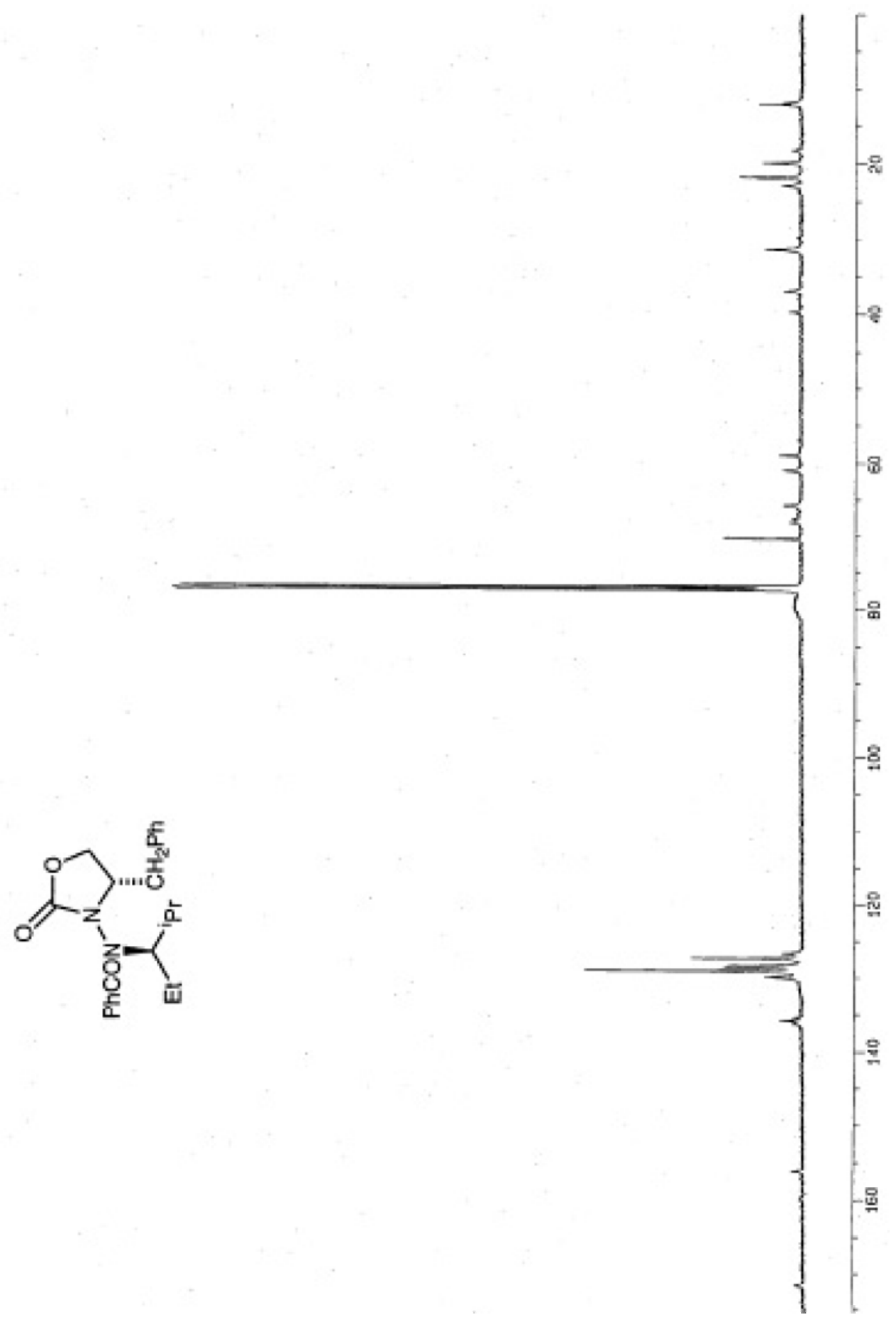




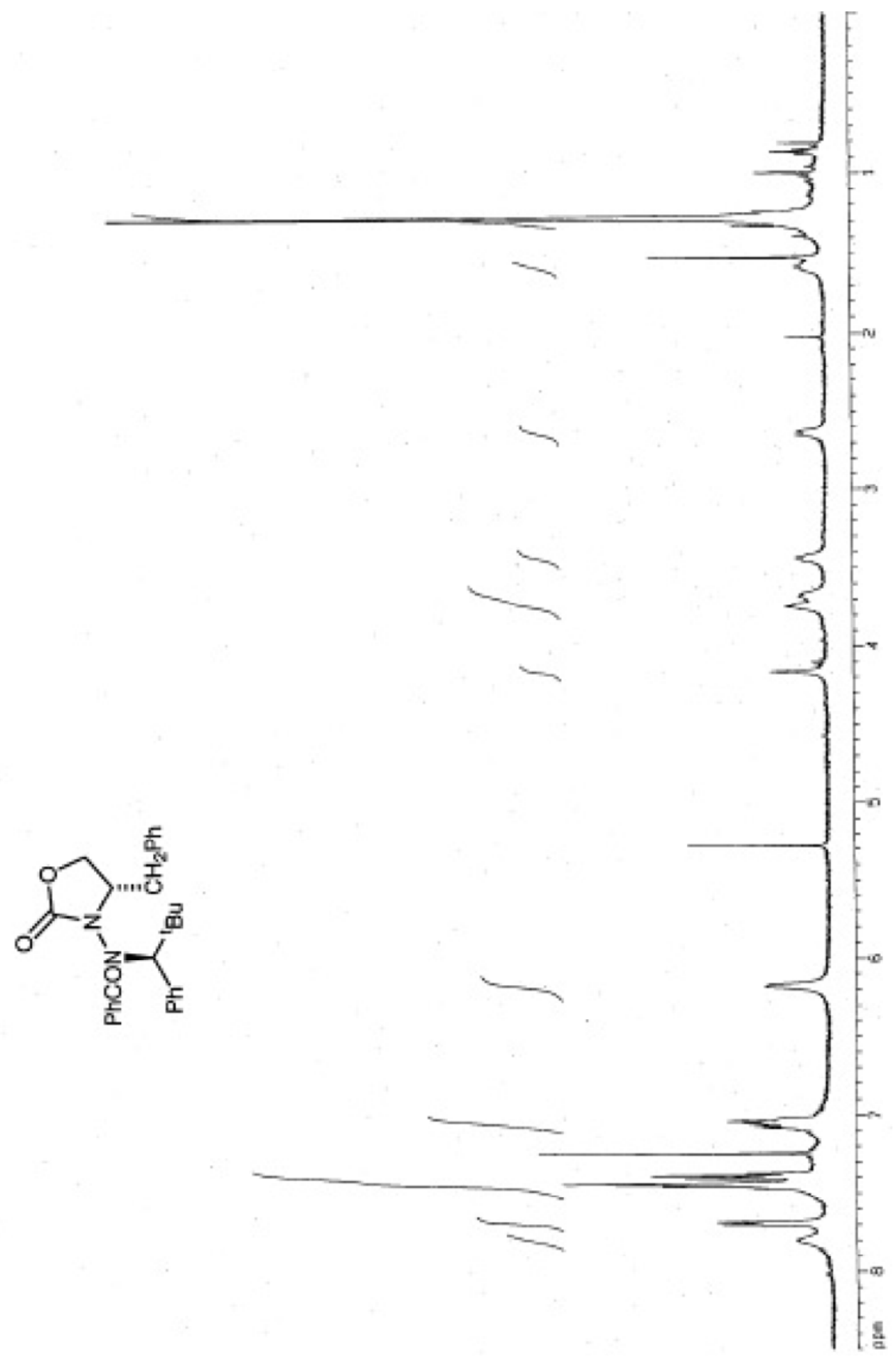




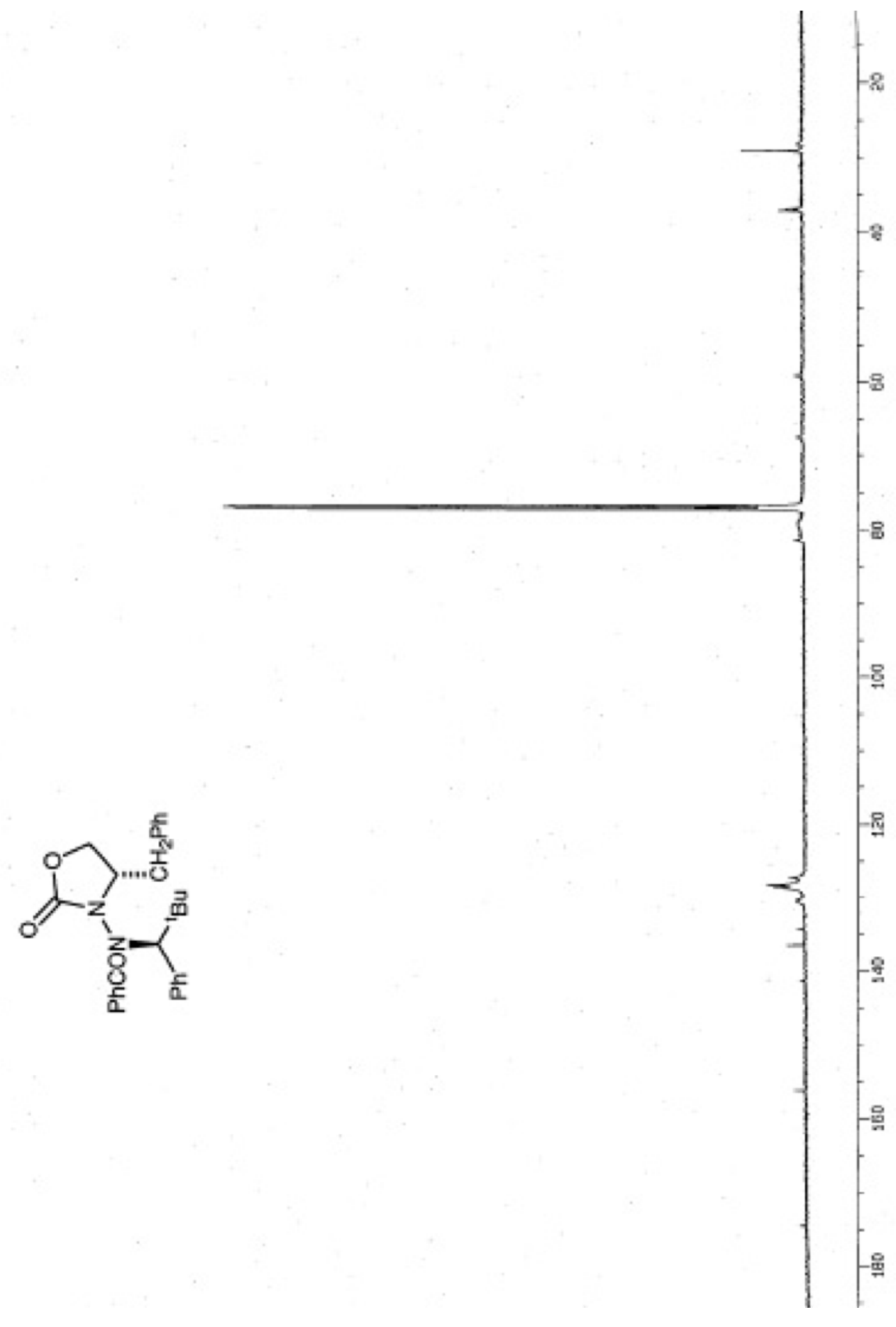

